\title{
Supporting Information（SI）
}

\section{Ag(I)-Catalyzed Tandem Reaction of Conjugated Ene-yne-ketones in the Presence of $\mathrm{PhI}(\mathrm{OAc})_{2}$ and TEA: Synthesis of 2-Alkenylfurans}

Shanjian Mao, Ling Tang, Chenggui Wu, Xianxia Tu, Qianwen Gao and Guisheng Deng*

Key Laboratory of Chemical Biology and Traditional Chinese Medicine Research (Ministry of Education of China), Hunan Normal University, Changsha 410081, China; Key Laboratory of the Assembly and Application of Organic Functional Molecules of Hunan Province, Hunan Normal University, Changsha 410081, China.

*E-mail: gsdeng@hunnu.edu.cn

\section{Content}

Experimental section .2

${ }^{1} \mathrm{H}$ and ${ }^{13} \mathrm{C}$ NMR spectra of $\mathbf{2 a - 2 n}, \mathbf{3 a - 3} \mathbf{c}$ 


\section{Experimental section}

General Methods. All reactions were carried out in a round flask with magnetic stirring. Unless otherwise noted, all reagents were purchased from Aladdin, Energy Chemical for direct use, or prepared as described in the literature. DCE was dried over $\mathrm{CaH}$ before use. For chromatographic purification, 200-300 mesh silica gel (Qingdao, China) was employed. For thin layer chromatography (TLC) analysis, Merck 25 TLC aluminium sheets (silica gel 60 GF254, $0.25 \mathrm{~mm}$ ) were used. Infrared spectra (IR) were recorded on a Bruker Vector 22 FT-IR spectrophotometer and are reported as wavelength numbers $\left(\mathrm{cm}^{-1}\right)$. Infrared spectra were recorded by preparing a $\mathrm{KBr}$ pellet containing the title compound. ${ }^{1} \mathrm{H}$ NMR and ${ }^{13} \mathrm{C}$ NMR were recorded on Bruker ARX $500 \mathrm{MHz}$ spectrometer in $\mathrm{CDCl}_{3}$ solution and the chemical shift were reported in parts per million $(\delta)$ relative to internal standard TMS $(0 \mathrm{ppm})$ and coupling constants are reported as Hertz $(\mathrm{Hz})$. Splitting patterns are designated as singlet (s), broad singlet (bs), doublet (d), triplet (t). Splitting patterns that could not be interpreted or easily visualized are designated as multiple (m). HRMS were performed on Thermo Scientific LTQ Qrbitrap XL mass spectrometer (ESI).

General Procedures. Synthesis of $2 \boldsymbol{a} . \mathrm{Et}_{3} \mathrm{~N}(0.3 \mathrm{mmol}, 30 \mathrm{mg})$ was syringed dropwise into a vigorous stirring mixture of 3-(non-2-yn-1-ylidene)pentane-2,4-dione 1a (0.2 mmol, $44 \mathrm{mg}), \mathrm{AgOAc}(0.04 \mathrm{mmol}, 6.7 \mathrm{mg})$ and $\mathrm{PhI}(\mathrm{OAc})_{2}(0.4 \mathrm{mmol}, 129$ $\mathrm{mg})$ in DCE $(2 \mathrm{~mL})$ at $60{ }^{\circ} \mathrm{C}$ under nitrogen atmosphere. After stirring for 3 hours, the reaction mixture was concentrated in vacuum, and the residue was purified by flash chromatography (eluted with petroleum ether/ethyl acetate $=15: 1$ ), providing product 2a.

1 mmol scale for one representative example: Synthesis of $2 a . \mathrm{Et}_{3} \mathrm{~N}(1.5 \mathrm{mmol}, 152$ $\mathrm{mg}$ ) was syringed dropwise into a vigorous stirring mixture of 3-(non-2-yn-1-ylidene)pentane-2,4-dione 1a (1 mmol, $220 \mathrm{mg})$, AgOAc (0.2 mmol, $33 \mathrm{mg})$ and $\mathrm{PhI}(\mathrm{OAc})_{2}(2 \mathrm{mmol}, 644 \mathrm{mg})$ in $\mathrm{DCE}(10 \mathrm{~mL})$ at $60{ }^{\circ} \mathrm{C}$ under nitrogen atmosphere. The mixture was stirred at $60{ }^{\circ} \mathrm{C}$ for another 5 hours, and then concentrated in vacuum. The residue was purified by column chromatography (eluted with petroleum ether/ethyl acetate $=15: 1)$, cleanly providing $\mathbf{2 a}(165 \mathrm{mg}, 75 \%)$.

\section{Control experiment}

Control Experiment (a): $\mathrm{Et}_{3} \mathrm{~N}(0.3 \mathrm{mmol}, 30 \mathrm{mg})$ was syringed dropwise into a vigorous stirring mixture of 3-(non-2-yn-1-ylidene)pentane-2,4-dione (1a $0.2 \mathrm{mmol}$, $44 \mathrm{mg}$ ), 1-methyl-4-vinylbenzene (0.2 mmol, 24mg), AgOAc (0.04 mmol, $6.7 \mathrm{mg})$, CuOTf $(0.04 \mathrm{mmol}, 9 \mathrm{mg})$ and $\mathrm{PhI}(\mathrm{OAc})_{2}(0.4 \mathrm{mmol}, 129 \mathrm{mg})$ in DCE $(2 \mathrm{~mL})$ at 60 ${ }^{\circ} \mathrm{C}$ under nitrogen atmosphere. After stirring for 3 hours, the reaction mixture was concentrated in vacuum, and the residue was purified by flash chromatography (eluted with petroleum ether/ethyl acetate $=15: 1$ ), providing product $\mathbf{2 a}$ in $78 \%$ yield.

Control Experiment (b): $\mathrm{Et}_{3} \mathrm{~N}(0.3 \mathrm{mmol}, 30 \mathrm{mg})$ was syringed dropwise into a vigorous stirring mixture of 3-(non-2-yn-1-ylidene)pentane-2,4-dione (1a $0.2 \mathrm{mmol}$, 
$44 \mathrm{mg})$, 4-methoxybenzaldehyde (0.2 mmol, 27mg), AgOAc (0.04 mmol, $6.7 \mathrm{mg})$, $\mathrm{Cu}(\mathrm{OTf})_{2}(0.04 \mathrm{mmol}, 14 \mathrm{mg})$ and $\mathrm{PhI}(\mathrm{OAc})_{2}(0.4 \mathrm{mmol}, 129 \mathrm{mg})$ in DCE $(2 \mathrm{~mL})$ at $60{ }^{\circ} \mathrm{C}$ under nitrogen atmosphere. After stirring for 3 hours, the reaction mixture was concentrated in vacuum, and the residue was purified by flash chromatography (eluted with petroleum ether/ethyl acetate $=15: 1$ ), providing product $\mathbf{2 a}$ in $77 \%$ yield.

Control Experiment (c): Methyl (E)-2-acetyl-5-cyclopropylpent-2-en-4-ynoate (0.2 mmol, 38mg) was dissolved in $\mathrm{MeOH}(1 \mathrm{~mL})$ and poured into vigorously stirred mixture of AgOAc (0.04 mmol, $6.7 \mathrm{mg}), \mathrm{MeONa}(0.4 \mathrm{mmol}, 22 \mathrm{mg})$ in $\mathrm{MeOH}(1 \mathrm{~mL})$ at $60{ }^{\circ} \mathrm{C}$ under nitrogen atmosphere. After stirring for 12 hours, no reaction occurred.

General Procedures. Synthesis of 3a: (Z)-6-cyclopropyl-3-methylhex-3-en-5-yn-2-one 1a (0.2 mmol, $30 \mathrm{mg})$ was dissolved in $\mathrm{MeOH}(1 \mathrm{~mL})$ and poured into vigorously stirred mixture of AgOAc $(0.04 \mathrm{mmol}$, $6.7 \mathrm{mg}), \mathrm{PhI}(\mathrm{OAc})_{2}(0.4 \mathrm{mmol}, 129 \mathrm{mg}), \mathrm{MeONa}(0.4 \mathrm{mmol}, 22 \mathrm{mg})$ in $\mathrm{MeOH}(1 \mathrm{~mL})$ at $60{ }^{\circ} \mathrm{C}$ under nitrogen atmosphere. After stirring for 6 hours, the reaction mixture was concentrated in vacuum, and the residue was purified by flash chromatography (eluted with petroleum ether/ethyl acetate $=10: 1$ ), providing product $\mathbf{3 a}$.

(E)-1-(5-(hept-1-en-1-yl)-2-methylfuran-3-yl)ethanone (2a).Yellow liquid (35 mg,

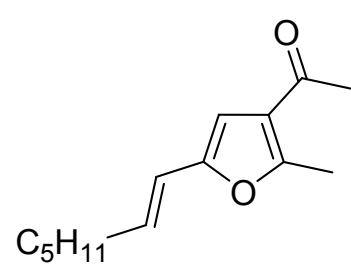
$80 \%), \mathrm{R}_{f}=0.5$ (PE:EA = 20:1). ${ }^{1} \mathrm{H}$ NMR $\left(500 \mathrm{MHz}, \mathrm{CDCl}_{3}\right.$, ppm) $\delta 6.29(\mathrm{~s}, 1 \mathrm{H}), 6.20-6.07(\mathrm{~m}, 2 \mathrm{H}), 2.56(\mathrm{~s}, 3 \mathrm{H}), 2.36(\mathrm{~s}$, $3 \mathrm{H}), 2.16(\mathrm{q}, J=7.0 \mathrm{~Hz}, 2 \mathrm{H}), 1.47-1.41(\mathrm{~m}, 2 \mathrm{H}), 1.34-1.29$ $(\mathrm{m}, 4 \mathrm{H}), 0.88(\mathrm{t}, J=7.0 \mathrm{~Hz}, 3 \mathrm{H}) .{ }^{13} \mathrm{C} \mathrm{NMR}(125.8 \mathrm{MHz}$, $\left.\mathrm{CDCl}_{3}, \mathrm{ppm}\right) \delta 194.2,157.3,151.0,131.2,122.6,117.6,105.9$, 32.8, 31.4, 29.1, 28.8, 22.5, 14.4, 14.0. IR (KBr): 2956, 2927, 2857, 1683, 1653, 1457, 1398, $950 \mathrm{~cm}^{-1}$. HRMS (ESI) $\mathrm{m} / z$ calcd for $\left[\mathrm{C}_{14} \mathrm{H}_{21} \mathrm{O}_{2}+\mathrm{H}\right]^{+}$ 221.1536 , found 221.1536 .

(E)-(5-(hept-1-en-1-yl)-2-methylfuran-3-yl)(phenyl)methanone (2b). Yellow liquid

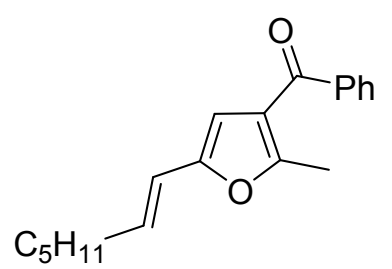

(43 mg, 76\%), $\mathrm{R}_{f}=0.55$ (PE:EA = 15:1). ${ }^{1} \mathrm{H}$ NMR (500 $\left.\mathrm{MHz}, \mathrm{CDCl}_{3}, \mathrm{ppm}\right) \delta 7.80-7.44(\mathrm{~m}, 5 \mathrm{H}), 6.28(\mathrm{~s}, 1 \mathrm{H})$, 6.24-6.10 (m, 2H), $2.53(\mathrm{~s}, 3 \mathrm{H}), 2.18(\mathrm{q}, J=7.0 \mathrm{~Hz}, 2 \mathrm{H})$, 1.49-1.43 (m, 2H), 1.34-1.30 (m, 4H), $0.90(\mathrm{t}, J=7.0 \mathrm{~Hz}$, $3 \mathrm{H}) .{ }^{13} \mathrm{C}$ NMR (125.8 MHz, $\left.\mathrm{CDCl}_{3}, \mathrm{ppm}\right) \delta 191.2,158.4$, $150.8,139.1,132.1,131.2,128.9,128.3,121.7,117.6,107.4$, 32.7, 31.3, 28.8, 22.5, 14.2, 14.0. IR (KBr): 2956, 2927, 2857, 1683, 1653, 1576, $1558,1539,1398,950 \mathrm{~cm}^{-1}$. HRMS (ESI) $\mathrm{m} / z$ calcd for $\left[\mathrm{C}_{19} \mathrm{H}_{23} \mathrm{O}_{2}+\mathrm{H}\right]^{+} 283.1693$, found 283.1693 .

(E)-1-(5-(hept-1-en-1-yl)-[2,2'-bifuran]-3-yl)ethanone (2c-1). Yellow liquid (39<smiles>Cc1oc(/C=C/CSc2ccccc2)cc1C(=O)c1ccco1</smiles>
$\mathrm{mg}, 72 \%), \mathrm{R}_{f}=0.55$ (PE:EA = 10:1). ${ }^{1} \mathrm{H}$ NMR $(500 \mathrm{MHz}$, $\left.\mathrm{CDCl}_{3}, \mathrm{ppm}\right) \delta 7.61(\mathrm{~d}, J=1.0 \mathrm{~Hz}, 1 \mathrm{H}), 7.25(\mathrm{~d}, J=3.5 \mathrm{~Hz}$, $1 \mathrm{H}), 6.70(\mathrm{~s}, 1 \mathrm{H}), 6.54(\mathrm{q}, J=3.5 \mathrm{~Hz}, 1 \mathrm{H}), 6.24-6.12(\mathrm{~m}$, $2 \mathrm{H}), 2.61(\mathrm{~s}, 3 \mathrm{H}), 2.20-2.15(\mathrm{~m}, 2 \mathrm{H}), 1.48-1.42(\mathrm{~m}, 2 \mathrm{H})$, 
1.33-1.30 (m, 4H), 0.89 (t, $J=7.0 \mathrm{~Hz}, 3 \mathrm{H}) .{ }^{13} \mathrm{C} \mathrm{NMR}\left(125.8 \mathrm{MHz}, \mathrm{CDCl}_{3}, \mathrm{ppm}\right) \delta$ 192.7, 176.9, 159.6, 153.7, 151.1, 145.8, 131.2, 117.9, 117.7, 112.2, 106.4, 32.8, 31.4, 28.8, 22.5, 14.5, 14.0. IR (KBr): 2956, 2927, 2857, 1683, 1653, 1647, 1398, $950 \mathrm{~cm}^{-1}$. HRMS (ESI) $m / z$ calcd for $\left[\mathrm{C}_{17} \mathrm{H}_{21} \mathrm{O}_{3}+\mathrm{H}\right]^{+} 273.1485$, found 273.1486 .

(Z)-1-(5-(hept-1-en-1-yl)-[2,2'-bifuran]-3-yl)ethanone (2c-2). Yellow liquid (39 mg,<smiles>CCCCC/C=C\c1cc(C(=O)c2ccco2)c(C)o1</smiles>
$72 \%), \mathrm{R}_{f}=0.55$ (PE:EA = 10: 1). ${ }^{1} \mathrm{H}$ NMR (500 MHz, $\mathrm{CDCl}_{3}$, ppm) $\delta 7.54((\mathrm{~d}, J=1.0 \mathrm{~Hz}, 1 \mathrm{H}), 7.25(\mathrm{~d}, J=3.5 \mathrm{~Hz}, 1 \mathrm{H}), 6.52(\mathrm{q}$, $J=3.5 \mathrm{~Hz}, 1 \mathrm{H}), 6.46(\mathrm{~s}, 1 \mathrm{H}), 6.38-6.31(\mathrm{~m}, 1 \mathrm{H}), 6.14(\mathrm{~d}, J=15.5$ $\mathrm{Hz}, 1 \mathrm{H}), 2.45(\mathrm{~s}, 3 \mathrm{H}), 2.20-2.15(\mathrm{~m}, 2 \mathrm{H}), 1.48-1.42(\mathrm{~m}, 2 \mathrm{H})$, $1.33-1.30(\mathrm{~m}, 4 \mathrm{H}), 0.89(\mathrm{t}, J=7.0 \mathrm{~Hz}, 3 \mathrm{H}) .{ }^{13} \mathrm{C}$ NMR $(125.8$ $\left.\mathrm{MHz}, \mathrm{CDCl}_{3}, \mathrm{ppm}\right) \delta 151.8,146.1,145.0,143.6,133.1,122.1,120.4,117.3,113.5$, 111.9, 107.5, 32.8, 29.4, 28.7, 22.5, 14.5, 14.0. IR (KBr): 2956, 2927, 2857, 1683, 1653, 1647, 1398, $950 \mathrm{~cm}^{-1}$. HRMS (ESI) $\mathrm{m} / z$ calcd for $\left[\mathrm{C}_{17} \mathrm{H}_{21} \mathrm{O}_{3}+\mathrm{H}\right]^{+} 273.1485$, found 273.1486 .

(E)-cyclopropyl(5-(hept-1-en-1-yl)-2-methylfuran-3-yl)methanone (2d-1). Yellow

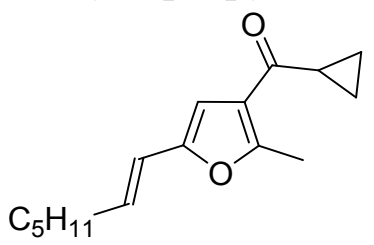
liquid (38 mg, 77\%), $\mathrm{R}_{f}=0.60$ (PE:EA = 20:1). ${ }^{1} \mathrm{H}$ NMR $(500$ $\left.\mathrm{MHz}, \mathrm{CDCl}_{3}, \mathrm{ppm}\right) \delta 6.45(\mathrm{~s}, 1 \mathrm{H}), 6.22-6.10(\mathrm{~m}, 2 \mathrm{H}), 2.58(\mathrm{~s}$, $3 \mathrm{H}), 2.30-2.25(\mathrm{~m}, 1 \mathrm{H}), 2.17(\mathrm{q}, J=7.0 \mathrm{~Hz}, 2 \mathrm{H}), 1.45-1.42$ $(\mathrm{m}, 2 \mathrm{H}), 1.35-1.30(\mathrm{~m}, 4 \mathrm{H}), 1.16-1.13(\mathrm{~m}, 2 \mathrm{H}), 0.95-0.88$ $(\mathrm{m}, 5 \mathrm{H}) .{ }^{13} \mathrm{C} \mathrm{NMR}\left(125.8 \mathrm{MHz}, \mathrm{CDCl}_{3}, \mathrm{ppm}\right) \delta 196.5,156.8$, $151.1,131.0,123.0,117.7,105.7,32.8,31.4,22.8,22.5,19.1,14.4,14.0,10.8$. IR (KBr): 2956, 2927, 2857, 1683, 1653, 1457, 1419, 1398, $950 \mathrm{~cm}^{-1}$. HRMS (ESI) $\mathrm{m} / z$ calcd for $\left[\mathrm{C}_{16} \mathrm{H}_{23} \mathrm{O}_{2}+\mathrm{H}\right]^{+}$247.1693, found 247.1693.

(Z)-cyclopropyl(5-(hept-1-en-1-yl)-2-methylfuran-3-yl)methanone (2d-2). Yellow

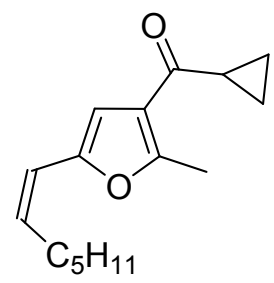
liquid (38 mg, 77\%), $\mathrm{R}_{f}=0.5(\mathrm{PE}: \mathrm{EA}=15: 1) .{ }^{1} \mathrm{H} \mathrm{NMR}(500 \mathrm{MHz}$, $\left.\mathrm{CDCl}_{3}, \mathrm{ppm}\right) \delta 6.28(\mathrm{~s}, 1 \mathrm{H}), 6.06-6.04(\mathrm{~m}, 2 \mathrm{H}), 2.83-2.77(\mathrm{~m}$, $1 \mathrm{H}), 2.40(\mathrm{~s}, 3 \mathrm{H}), 2.16-2.12(\mathrm{~m}, 2 \mathrm{H}), 1.46-1.40(\mathrm{~m}, 2 \mathrm{H})$, $1.33-1.28(\mathrm{~m}, 4 \mathrm{H}), 1.11-1.04(\mathrm{~m}, 4 \mathrm{H}), 0.89(\mathrm{t}, J=7.0 \mathrm{~Hz}, 3 \mathrm{H}) .{ }^{13} \mathrm{C}$ NMR (125.8 MHz, $\left.\mathrm{CDCl}_{3}, \mathrm{ppm}\right) \delta 196.5,156.8,151.1,131.0$, $122.9,117.7,105.7,32.8,31.4,28.9,22.5,19.1,14.4,14.0,10.8$. IR (KBr): 2956, 2927, 2857, 1683, 1653, 1457, 1419, 1398, $950 \mathrm{~cm}^{-1}$. HRMS (ESI) $m / z$ calcd for $\left[\mathrm{C}_{16} \mathrm{H}_{23} \mathrm{O}_{2}+\mathrm{H}\right]^{+} 247.1693$, found 247.1692 .

(E)-methyl 5-(hept-1-en-1-yl)-2-methylfuran-3-carboxylate (2e). Yellow liquid (36

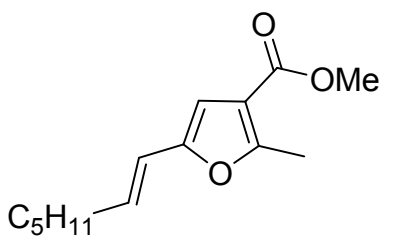
$\mathrm{mg}, 77 \%), \mathrm{R}_{f}=0.65$ (PE:EA = 20:1). ${ }^{1} \mathrm{H}$ NMR $(500 \mathrm{MHz}$, $\left.\mathrm{CDCl}_{3}, \mathrm{ppm}\right) \delta 6.32(\mathrm{~s}, 1 \mathrm{H}), 6.18-6.07(\mathrm{~m}, 2 \mathrm{H}), 3.79(\mathrm{~s}, 3 \mathrm{H})$, $2.55(\mathrm{~s}, 3 \mathrm{H}), 2.15(\mathrm{q}, J=7.0 \mathrm{~Hz}, 2 \mathrm{H}), 1.45-1.41(\mathrm{~m}, 2 \mathrm{H})$, $1.33-1.28(\mathrm{~m}, 4 \mathrm{H}), 0.89(\mathrm{t}, J=7.0 \mathrm{~Hz}, 3 \mathrm{H}) .{ }^{13} \mathrm{C} \mathrm{NMR}(125.8$ $\left.\mathrm{MHz}, \mathrm{CDCl}_{3}, \mathrm{ppm}\right) \delta 164.5,158.1,151.1,130.9,117.7$, $114.5,106.1,51.2,32.7,31.4,28.9,22.5,14.0,13.8$. IR (KBr): 2956, 2927, 2857 , 1683, 1653, 1457, 1398, $950 \mathrm{~cm}^{-1}$. HRMS (ESI) $\mathrm{m} / z$ calcd for $\left[\mathrm{C}_{14} \mathrm{H}_{21} \mathrm{O}_{3}+\mathrm{H}\right]^{+}$ 
(E)-tert-butyl 5-(hept-1-en-1-yl)-2-methylfuran-3-carboxylate (2f). Yellow liquid

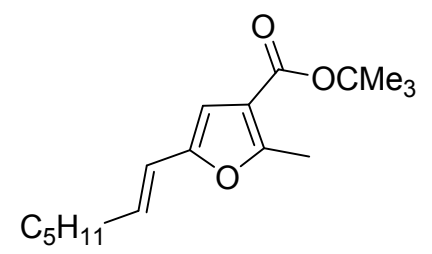
(45 mg, 81\%), $\mathrm{R}_{f}=0.5$ (PE:EA = 20:1). ${ }^{1} \mathrm{H}$ NMR (500 $\left.\mathrm{MHz}, \mathrm{CDCl}_{3}, \mathrm{ppm}\right) \delta 6.29(\mathrm{~s}, 1 \mathrm{H}), 6.17-6.06(\mathrm{~m}, 2 \mathrm{H}), 2.53$ $(\mathrm{s}, 3 \mathrm{H}), 2.15(\mathrm{q}, J=6.8 \mathrm{~Hz}, 2 \mathrm{H}), 1.54(\mathrm{~s}, 9 \mathrm{H}), 1.46-1.41$ (m, 2H), 1.33-1.29 (m, 4H), 0.89 (t, $J=6.8 \mathrm{~Hz}, 3 \mathrm{H}) .{ }^{13} \mathrm{C}$ NMR $\left(125.8 \mathrm{MHz}, \mathrm{CDCl}_{3}, \mathrm{ppm}\right) \delta 163.5,157.3,150.8$, $130.5,117.8,116.2,106.5,80.4,32.7,31.4,28.8,28.3,22.5,14.0,13.8$. IR (KBr): 2956, 2927, 2857, 1683, 1653, 1457, 1398, $950 \mathrm{~cm}^{-1}$. HRMS (ESI) $\mathrm{m} / z$ calcd for $\left[\mathrm{C}_{17} \mathrm{H}_{27} \mathrm{O}_{3}+\mathrm{H}\right]^{+} 279.1955$, found 279.1952 .

(E)-allyl 5-(hept-1-en-1-yl)-2-methylfuran-3-carboxylate (2g). Yellow liquid (41 mg, 79\%), $\mathrm{R}_{f}=0.65$ (PE:EA = 20:1). ${ }^{1} \mathrm{H}$ NMR $(500 \mathrm{MHz}$,<smiles>C=CCOC(=O)c1cc(/C=C/[SbH2])oc1C</smiles>
$\left.\mathrm{CDCl}_{3}, \mathrm{ppm}\right) \delta 6.35(\mathrm{~s}, 1 \mathrm{H}), 6.19-6.07 \quad(\mathrm{~m}, 2 \mathrm{H})$, $6.02-5.94(\mathrm{~m}, 1 \mathrm{H}), 5.35(\mathrm{~d}, J=17.2 \mathrm{~Hz}, 1 \mathrm{H}), 5.25(\mathrm{~d}, J=$ $10.4 \mathrm{~Hz}, 1 \mathrm{H}), 4.72(\mathrm{~d}, J=5.6 \mathrm{~Hz}, 2 \mathrm{H}), 2.57(\mathrm{~s}, 3 \mathrm{H}), 2.16$ $(\mathrm{q}, J=7.0 \mathrm{~Hz}, 2 \mathrm{H}), 1.47-1.41(\mathrm{~m}, 2 \mathrm{H}), 1.34-1.28(\mathrm{~m}$, $4 \mathrm{H}), 0.89(\mathrm{t}, J=7.0 \mathrm{~Hz}, 3 \mathrm{H}) .{ }^{13} \mathrm{C}$ NMR $(125.8 \mathrm{MHz}$, $\left.\mathrm{CDCl}_{3}, \mathrm{ppm}\right) \delta 163.7,158.3,151.2,132.4,130.9,117.9,117.7,114.5,106.2,64.7$, 32.8, 31.4, 28.8, 22.5, 14.0. IR (KBr): 2956, 2927, 2857, 1683, 1653, 1583, 1457, 1398, $950 \mathrm{~cm}^{-1}$. HRMS (ESI) $\mathrm{m} / z$ calcd for $\left[\mathrm{C}_{16} \mathrm{H}_{23} \mathrm{O}_{3}+\mathrm{H}\right]^{+}$263.1642, found 263.1642 .

(E)-benzyl 5-(hept-1-en-1-yl)-2-methylfuran-3-carboxylate (2h). Yellow liquid (50

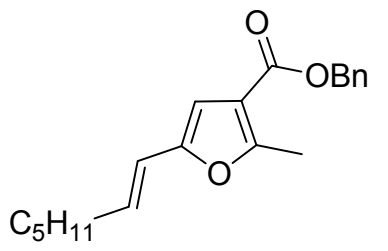
$\mathrm{mg}, 80 \%), \mathrm{R}_{f}=0.68(\mathrm{PE}: \mathrm{EA}=20: 1) .{ }^{1} \mathrm{H}$ NMR $(500 \mathrm{MHz}$, $\left.\mathrm{CDCl}_{3}, \mathrm{ppm}\right) \delta 7.41-7.31(\mathrm{~m}, 5 \mathrm{H}), 6.37(\mathrm{~s}, 1 \mathrm{H}), 6.19-6.08(\mathrm{~m}$, $2 \mathrm{H}), 5.27(\mathrm{~s}, 2 \mathrm{H}), 2.57(\mathrm{~s}, 3 \mathrm{H}), 2.16(\mathrm{q}, J=7.0 \mathrm{~Hz}, 2 \mathrm{H})$, $1.46-1.42(\mathrm{~m}, 2 \mathrm{H}), 1.34-1.26(\mathrm{~m}, 4 \mathrm{H}), 0.9$ (t, $J=7.0 \mathrm{HZ}$, $3 \mathrm{H}) .{ }^{13} \mathrm{C}$ NMR $\left(125.8 \mathrm{MHz}, \mathrm{CDCl}_{3}, \mathrm{ppm}\right) \delta 163.9,158.4$, $151.2,136.3,130.9,128.6,128.1,128.0,117.7,114.5,106.2,65.8,32.7,31.4,28.8$, 22.6, 14.1, 13.9. IR (KBr): 2956, 2927, 2857, 1683, 1653, 1583, 1558, 1539, 1398, $950 \mathrm{~cm}^{-1}$. HRMS (ESI) $\mathrm{m} / z$ calcd for $\left[\mathrm{C}_{20} \mathrm{H}_{25} \mathrm{O}_{3}+\mathrm{H}\right]^{+} 313.1798$, found 313.1799 .

(E)-5-(hept-1-en-1-yl)-2-methyl- $N$-phenylfuran-3-carboxamide (2i). Yellow liquid<smiles>Cc1oc(/C=C/[SbH2])cc1C(=O)Nc1ccccc1</smiles>
(40 mg, 67\%), $\mathrm{R}_{f}=0.50$ (PE:EA = 15:1). ${ }^{1} \mathrm{H}$ NMR $(500$ $\left.\mathrm{MHz}, \mathrm{CDCl}_{3}, \mathrm{ppm}\right) \delta 7.56(\mathrm{~d}, J=7.8 \mathrm{~Hz}, 2 \mathrm{H}), 7.38(\mathrm{~s}, 1 \mathrm{H})$, $7.34(\mathrm{t}, J=7.8 \mathrm{~Hz}, 2 \mathrm{H}), 7.12(\mathrm{t}, J=7.8 \mathrm{~Hz}, 1 \mathrm{H}), 6.24-6.10$ $(\mathrm{m}, 3 \mathrm{H}), 2.62(\mathrm{~s}, 3 \mathrm{H}), 2.20(\mathrm{q}, J=7.0 \mathrm{~Hz}, 2 \mathrm{H}), 1.49-1.44(\mathrm{~m}$, $2 \mathrm{H}), 1.34-1.32(\mathrm{~m}, 4 \mathrm{H}), 0.91(\mathrm{t}, J=7.0 \mathrm{~Hz}, 3 \mathrm{H}) .{ }^{13} \mathrm{C} \mathrm{NMR}$ (125.8 MHz, $\left.\mathrm{CDCl}_{3}, \mathrm{ppm}\right) \delta 162.1,156.8,151.4,137.8,131.6,129.0,124.3,120.1$, 117.6, 117.0, 103.5, 32.8, 31.4, 28.8, 22.5, 14.0, 13.7. IR (KBr): 2956, 2927, 2857, $1680,1653,1558,1539,1419,950,668 \mathrm{~cm}^{-1}$. HRMS (ESI) $\mathrm{m} / z$ calcd for $\left[\mathrm{C}_{19} \mathrm{H}_{24} \mathrm{NO}_{2}\right.$ 
$+\mathrm{H}]^{+}$298.1802, found 298.1801.

(E)-5-(hept-1-en-1-yl)-2-methyl- $N$-phenylfuran-3-carboxamide (2j). Yellow liquid<smiles>Cc1oc(/C=C/[B]c2ccccc2)cc1C(=O)NOCCO</smiles>
(43 mg, 72\%), $\mathrm{R}_{f}=0.50$ (PE:EA $\left.=15: 1\right) .{ }^{1} \mathrm{H}$ NMR (500 $\left.\mathrm{MHz} \mathrm{CDCl}_{3}, \mathrm{ppm}\right) \delta 7.44(\mathrm{~d}, J=8.2 \mathrm{~Hz}, 2 \mathrm{H}), 7.31(\mathrm{~s}$, $1 \mathrm{H}), 7.13(\mathrm{~d}, J=8.2 \mathrm{~Hz}, 2 \mathrm{H}), 6.24-6.09(\mathrm{~m}, 3 \mathrm{H}), 2.62(\mathrm{~s}$, $3 \mathrm{H}), 2.32(\mathrm{~s}, 3 \mathrm{H}), 2.18(\mathrm{q}, J=7.0 \mathrm{~Hz}, 2 \mathrm{H}), 1.49-1.43(\mathrm{~m}$, $2 \mathrm{H}), 1.34-1.31(\mathrm{~m}, 4 \mathrm{H}), 0.90(\mathrm{t}, J=7.0 \mathrm{~Hz}, 3 \mathrm{H}) .{ }^{13} \mathrm{C}$ NMR (125.8 MHz, $\mathrm{CDCl}_{3}$, ppm) $\delta 162.0,156.6,151.3,135.2,133.9,131.5,129.5$, 120.2, 117.6, 117.1, 103.6, 32.8, 31.4, 28.8, 22.5, 20.8, 14.0, 13.7. IR (KBr): 2956, 2927, 2857, 1680, 1653, 1558, 1539, 1419, 950,786 $\mathrm{cm}^{-1}$. HRMS (ESI) $\mathrm{m} / z$ calcd for $\left[\mathrm{C}_{20} \mathrm{H}_{26} \mathrm{NO}_{2}+\mathrm{H}\right]^{+}$312.1958, found 312.1956.

(E)- $N$-(4-chlorophenyl)-5-(hept-1-en-1-yl)-2-methylfuran-3-carboxamide (2k). Yellow liquid (50 mg, 76\%), $\mathrm{R}_{f}=0.50$ (PE:EA = 15:1). ${ }^{1} \mathrm{H}$ NMR $\left(500 \mathrm{MHz}, \mathrm{CDCl}_{3}, \mathrm{ppm}\right) \delta 7.52(\mathrm{~d}, J=8.8 \mathrm{~Hz}$, $2 \mathrm{H}), 7.36(\mathrm{~s}, 1 \mathrm{H}), 7.29(\mathrm{~d}, J=8.8 \mathrm{~Hz}, 2 \mathrm{H}), 6.25-6.08(\mathrm{~m}$, $3 \mathrm{H}), 2.62(\mathrm{~s}, 3 \mathrm{H}), 2.18(\mathrm{t}, J=7.0 \mathrm{~Hz}, 2 \mathrm{H}), 1.49-1.43(\mathrm{~m}$, $\mathrm{C}_{5} \mathrm{H}_{11}$ $2 \mathrm{H}), 1.34-1.31(\mathrm{~m}, 4 \mathrm{H}), 0.90(\mathrm{t}, J=7.0 \mathrm{~Hz}, 3 \mathrm{H}) .{ }^{13} \mathrm{C}$ NMR (125.8 MHz, $\mathrm{CDCl}_{3}$, ppm) $\delta 162.0,157.0,151.4,136.4,131.8,129.2,129.0$, 103.3, 32.8, 31.4, 28.8, 22.6, 14.1, 13.8. IR (KBr): 2956, 2927, 2857, 1680, 1653, $1583,1539,1419,950,786 \mathrm{~cm}^{-1}$. HRMS (ESI) $\mathrm{m} / z$ calcd for $\left[\mathrm{C}_{19} \mathrm{H}_{23} \mathrm{ClNO}_{2}+\mathrm{H}\right]^{+}$ 332.1412 , found 332.1412 .

(E)-5-(hept-1-en-1-yl)-2-methyl-3-(phenylsulfonyl)furan (21-1). Yellow liquid (48

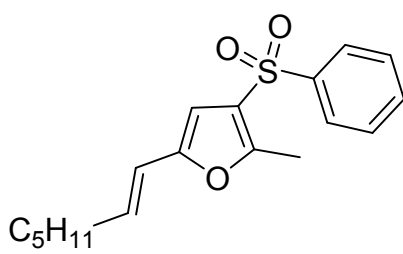
$\mathrm{mg}, 76 \%), \mathrm{R}_{f}=0.60$ (PE:EA = 20:1). ${ }^{1} \mathrm{H}$ NMR $(500 \mathrm{MHz}$, $\left.\mathrm{CDCl}_{3}, \mathrm{ppm}\right) \delta 7.92-7.47(\mathrm{~m}, 5 \mathrm{H}), 6.25(\mathrm{~s}, 1 \mathrm{H}), 6.19-6.13$ $(\mathrm{m}, 1 \mathrm{H}), 6.04-6.00(\mathrm{~m}, 1 \mathrm{H}), 2.56(\mathrm{~s}, 3 \mathrm{H}), 2.12(\mathrm{q}, J=7.0$ $\mathrm{Hz}, 2 \mathrm{H}), 1.45-1.38(\mathrm{~m}, 2 \mathrm{H}), 1.32-1.25(\mathrm{~m}, 4 \mathrm{H}), 0.87(\mathrm{t}, J=$ $7.0 \mathrm{~Hz}, 3 \mathrm{H}) .{ }^{13} \mathrm{C} \mathrm{NMR}\left(125.8 \mathrm{MHz}, \mathrm{CDCl}_{3}, \mathrm{ppm}\right) \delta 155.1$, $152.1,142.6,134.2,133.0,129.2,126.8,123.9,107.7,32.7,31.5,28.9,22.5,14.0,3.0$. IR (KBr): 2956, 2927, 2857, 1653, 1558, 1539, 1398, $950 \mathrm{~cm}^{-1}$. HRMS (ESI) $\mathrm{m} / z$ calcd for $\left[\mathrm{C}_{18} \mathrm{H}_{23} \mathrm{O}_{3} \mathrm{~S}+\mathrm{H}\right]^{+}$319.1362, found 319.1363.

(Z)-5-(hept-1-en-1-yl)-2-methyl-3-(phenylsulfonyl)furan (2l-2). Yellow liquid (48

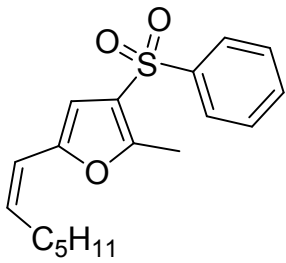
$\mathrm{mg}, 76 \%), \mathrm{R}_{f}=0.60(\mathrm{PE}: \mathrm{EA}=20: 1) .{ }^{1} \mathrm{H} \mathrm{NMR}\left(500 \mathrm{MHz}, \mathrm{CDCl}_{3}\right.$, ppm) $\delta 7.92-7.47(\mathrm{~m}, 5 \mathrm{H}), 6.38(\mathrm{~s}, 1 \mathrm{H}), 6.04-6.00(\mathrm{~m}, 1 \mathrm{H})$, 5.64-5.59 (m, 1H), $2.56(\mathrm{~s}, 3 \mathrm{H}), 2.34-2.30(\mathrm{~m}, 2 \mathrm{H}), 1.45-1.37$ $(\mathrm{m}, 2 \mathrm{H}), 1.32-1.25(\mathrm{~m}, 4 \mathrm{H}), 0.87(\mathrm{t}, J=7.0 \mathrm{~Hz}, 3 \mathrm{H}) .{ }^{13} \mathrm{C} \mathrm{NMR}$ $\left(125.8 \mathrm{MHz}, \mathrm{CDCl}_{3}, \mathrm{ppm}\right) \delta 154.9,151.9,133.1,129.3,126.9$, 123.8, 116.9, 112.9, 104.9, 31.5, 29.3, 28.6, 22.5, 14.0, 13.0. IR (KBr): 2956, 2927, $2857,1653,1558,1539,1398,950 \mathrm{~cm}^{-1}$. HRMS (ESI) $m / z$ calcd for $\left[\mathrm{C}_{18} \mathrm{H}_{23} \mathrm{O}_{3} \mathrm{~S}+\mathrm{H}\right]^{+}$ 319.1362 , found 319.1363 . 
(E)-5-(hept-1-en-1-yl)-2-methyl-3-tosylfuran (2m-1). Yellow liquid (53 mg, 80\%),

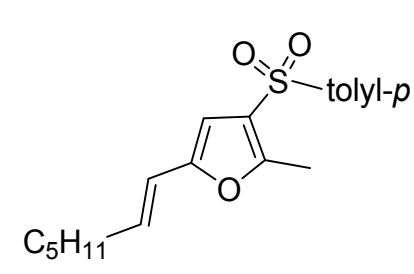
$\mathrm{R}_{f}=0.60$ (PE:EA = 20:1). ${ }^{1} \mathrm{H} \mathrm{NMR}\left(500 \mathrm{MHz}, \mathrm{CDCl}_{3}\right.$, ppm) $\delta 7.81-7.26(\mathrm{~m}, 4 \mathrm{H}), 6.24(\mathrm{~s}, 1 \mathrm{H}), 6.19-6.13(\mathrm{~m}, 1 \mathrm{H})$, $6.03(\mathrm{~d}, J=7.0 \mathrm{~Hz}, 1 \mathrm{H}), 2.55(\mathrm{~s}, 3 \mathrm{H}), 2.40(\mathrm{~s}, 3 \mathrm{H}), 2.13(\mathrm{q}$, $J=7.0 \mathrm{~Hz}, 2 \mathrm{H}), 1.46-1.39(\mathrm{~m}, 2 \mathrm{H}), 1.32-1.27(\mathrm{~m}, 4 \mathrm{H})$, $0.87(\mathrm{t}, J=7.0 \mathrm{~Hz}, 3 \mathrm{H}) .{ }^{13} \mathrm{C} \mathrm{NMR}\left(125.8 \mathrm{MHz}, \mathrm{CDCl}_{3}, \mathrm{ppm}\right)$ $\delta 154.8,151.9,144.0,139.7,132.6,129.9,126.9,124.23$, 117.1, 107.7, 32.7, 31.5, 29.3, 22.5, 21.6, 14.0, 13.0. IR (KBr): 2956, 2927, 2857, 1653, 1558, 1539, 1398, $950 \mathrm{~cm}^{-1}$. HRMS (ESI) $\mathrm{m} / z$ calcd for $\left[\mathrm{C}_{19} \mathrm{H}_{25} \mathrm{O}_{3} \mathrm{~S}+\mathrm{H}\right]^{+}$ 333.1519 , found 333.1519 .

(Z)-5-(hept-1-en-1-yl)-2-methyl-3-tosylfuran (2m-2). Yellow liquid (53 mg, 80\%),<smiles>CCCCCC/C=C\c1cc(S(=O)(=O)O[Na])c(C)o1</smiles>
$\mathrm{R}_{f}=0.60(\mathrm{PE}: \mathrm{EA}=20: 1) .{ }^{1} \mathrm{H}$ NMR $\left(500 \mathrm{MHz}, \mathrm{CDCl}_{3}, \mathrm{ppm}\right) \delta$ $7.81-7.26(\mathrm{~m}, 4 \mathrm{H}), 6.37(\mathrm{~s}, 1 \mathrm{H}), 6.03(\mathrm{~d}, J=7.0 \mathrm{~Hz}, 1 \mathrm{H})$, 5.64-5.59 (m, 1H), $2.56(\mathrm{~s}, 3 \mathrm{H}), 2.40(\mathrm{~s}, 3 \mathrm{H}), 2.32(\mathrm{q}, J=7.0 \mathrm{~Hz}$, $2 \mathrm{H}), 1.46-1.39(\mathrm{~m}, 2 \mathrm{H}), 1.32-1.27(\mathrm{~m}, 4 \mathrm{H}), 0.87$ (t, $J=7.0 \mathrm{~Hz}$, $3 \mathrm{H}) .{ }^{13} \mathrm{C} \mathrm{NMR}\left(125.8 \mathrm{MHz}, \mathrm{CDCl}_{3}, \mathrm{ppm}\right) \delta 154.6,151.8,143.9$, $139.7,134.1,129.8,126.9,124.2,115.9,104.9,31.3,28.9,28.7$, 22.1, 19.3, 14.0, 13.0. IR (KBr): 2956, 2927, 2857, 1653, 1558, 1539, 1398, $950 \mathrm{~cm}^{-1}$. HRMS (ESI) $m / z$ calcd for $\left[\mathrm{C}_{19} \mathrm{H}_{25} \mathrm{O}_{3} \mathrm{~S}+\mathrm{H}\right]^{+} 333.1519$, found 333.1519 .

(E)-(5-(hept-1-en-1-yl)-2-phenylfuran-3-yl)(phenyl)methanone (2n). Yellow liquid<smiles>O=C(c1ccccc1)c1cc(/C=C/c2ccccc2)oc1-c1ccccc1</smiles>

(33 mg, 48\%), $\mathrm{R}_{f}=0.5$ (PE:EA = 15:1). ${ }^{1} \mathrm{H}$ NMR $(500 \mathrm{MHz}$, $\left.\mathrm{CDCl}_{3}, \mathrm{ppm}\right) \delta 7.86-7.26(\mathrm{~m}, 10 \mathrm{H}), 6.41-6.35(\mathrm{~m}, 2 \mathrm{H}), 6.23$ $(\mathrm{d}, J=15.5 \mathrm{~Hz}, 1 \mathrm{H}), 2.23(\mathrm{q}, J=7.0 \mathrm{~Hz}, 2 \mathrm{H}), 1.53-1.47(\mathrm{~m}$, $2 \mathrm{H}), 1.36-1.34(\mathrm{~m}, 4 \mathrm{H}), 0.92(\mathrm{t}, J=7.0 \mathrm{~Hz}, 3 \mathrm{H}) .{ }^{13} \mathrm{C} \mathrm{NMR}$ $\left(125.8 \mathrm{MHz}, \mathrm{CDCl}_{3}, \mathrm{ppm}\right) \delta 191.8,154.4,151.8,138.1,137.5$, $132.8,132.6,130.2,129.8,129.7,128.8,128.3,128.3,127.4,122.4,117.6,109.6$, 32.9, 31.4, 28.8, 22.6, 14.1. IR (KBr): 2956, 2927, 2857, 1683, 1653, 1583, 1576, $1558,1539,1506,950 \mathrm{~cm}^{-1}$. HRMS (ESI) $\mathrm{m} / z$ calcd for $\left[\mathrm{C}_{24} \mathrm{H}_{25} \mathrm{O}_{2}+\mathrm{H}\right]^{+} 345.1849$, found 345.1850 .

1-(5-(cyclohexylidenemethyl)-2-methylfuran-3-yl)ethanone (2o). Yellow liquid (60

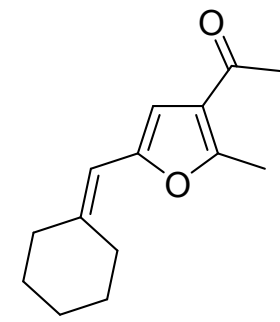

219.1380 . $\mathrm{mg}, 70 \%), \mathrm{R}_{f}=0.6$ (PE:EA = 15:1). ${ }^{1} \mathrm{H} \mathrm{NMR}\left(500 \mathrm{MHz}, \mathrm{CDCl}_{3}\right.$, ppm) $\delta 6.33(\mathrm{~s}, 1 \mathrm{H}), 5.86(\mathrm{~s}, 1 \mathrm{H}), 2.56(\mathrm{~s}, 3 \mathrm{H}), 2.53(\mathrm{t}, J=5.8 \mathrm{~Hz}$, $2 \mathrm{H}), 2.38(\mathrm{~s}, 3 \mathrm{H}), 2.21(\mathrm{t}, J=5.8 \mathrm{~Hz}, 2 \mathrm{H}), 1.62-1.57(\mathrm{~m}, 6 \mathrm{H}) .{ }^{13} \mathrm{C}$ NMR (125.8 MHz, $\left.\mathrm{CDCl}_{3}, \mathrm{ppm}\right) \delta 194.3,156.5,151.0,144.8$, $122.5,110.5,107.5,37.6,30.2,29.1,28.5,27.5,26.4,14.5$. IR (KBr): 3444, 2928, 2855, 2361, 2335, 1676, 1645, 1455, 751950 $\mathrm{cm}^{-1}$. HRMS (ESI) $\mathrm{m} / z$ calcd for $\left[\mathrm{C}_{14} \mathrm{H}_{18} \mathrm{O}_{2}+\mathrm{H}\right]^{+} 219.1389$, found 
(5-(cyclohexylidenemethyl)-2-methylfuran-3-yl)(phenyl)methanone (2p). Yellow liquid (58 mg, 52\%), $\mathrm{R}_{f}=0.4$ (PE:EA = 15:1). ${ }^{1} \mathrm{H}$ NMR $(500$

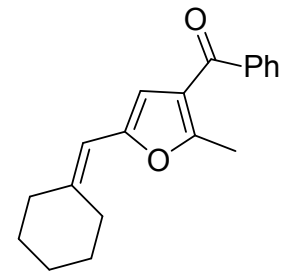
$\left.\mathrm{MHz}, \mathrm{CDCl}_{3}, \mathrm{ppm}\right) \delta 7.82-7.78(\mathrm{~m}, 2 \mathrm{H}), 7.57-7.53(\mathrm{~m}, 1 \mathrm{H}), 7.49$ $7.45(\mathrm{~m}, 2 \mathrm{H}), 6.32(\mathrm{~s}, 1 \mathrm{H}), 5.91(\mathrm{~s}, 1 \mathrm{H}), 2.57-2.54(\mathrm{~m}, 2 \mathrm{H}), 2.51$ $(\mathrm{s}, 3 \mathrm{H}), 2.23(\mathrm{t}, J=5.7 \mathrm{~Hz}, 2 \mathrm{H}), 1.63-1.59(\mathrm{~m}, 6 \mathrm{H}) .{ }^{13} \mathrm{C} \mathrm{NMR}$ $\left(125.8 \mathrm{MHz}, \mathrm{CDCl}_{3}, \mathrm{ppm}\right) \delta 194.2,154.3,152.1,146.1,130.2$, $129.4,128.3,128.2,123.5,110.1,109.7,37.7,30.4,29.8,28.6$, 27.6, 26.4. IR (KBr): 3442, 2926, 2361, 2335, 1867, 1740, 1646, 1515, 1454, 725 $\mathrm{cm}^{-1}$. HRMS (ESI) $\mathrm{m} / z$ calcd for $\left[\mathrm{C}_{19} \mathrm{H}_{20} \mathrm{O}_{2}+\mathrm{H}\right]^{+} 281.1539$, found 281.1536.

(5-(cyclohexylidenemethyl)-2-methylfuran-3-yl)(cyclopropyl)methanone (2q-1). Yellow liquid (66 mg, 68\%), $\mathrm{R}_{f}=0.6$ (PE:EA = 15:1). ${ }^{1} \mathrm{H}$ NMR

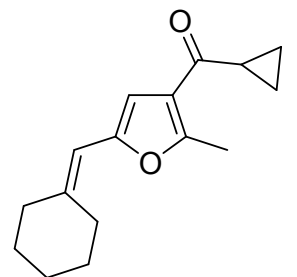
$\left(500 \mathrm{MHz}, \mathrm{CDCl}_{3}, \mathrm{ppm}\right) \delta 6.48(\mathrm{~s}, 1 \mathrm{H}), 5.89(\mathrm{~s}, 1 \mathrm{H}), 2.58(\mathrm{~s}, 3 \mathrm{H})$, $2.57-2.54(\mathrm{~m}, 2 \mathrm{H}), 2.31-2.27(\mathrm{~m}, 1 \mathrm{H}), 2.22(\mathrm{t}, J=5.7 \mathrm{~Hz}, 2 \mathrm{H})$, $1.62-1.58(\mathrm{~m}, 6 \mathrm{H}), 1.17-1.13(\mathrm{~m}, 2 \mathrm{H}), 0.95-0.91(\mathrm{~m}, 2 \mathrm{H}) .{ }^{13} \mathrm{C}$ NMR (125.8 MHz, $\left.\mathrm{CDCl}_{3}, \mathrm{ppm}\right) \delta 196.6,156.0,151.0,144.6$, $122.8,110.3,107.3,37.6,30.2,28.5,27.5,26.4,19.1,14.5,10.9$. IR (KBr): 3442, 2926, 2361, 2335, 1646, 1515, 1454, $725 \mathrm{~cm}^{-1}$. HRMS (ESI) $\mathrm{m} / \mathrm{z}$ calcd for $\left[\mathrm{C}_{16} \mathrm{H}_{20} \mathrm{O}_{2}+\mathrm{H}\right]^{+}$245.1537, found 245.1538.

1-(5-(cyclohexylidenemethyl)-2-cyclopropylfuran-3-yl)ethanone (2q-2). Yellow

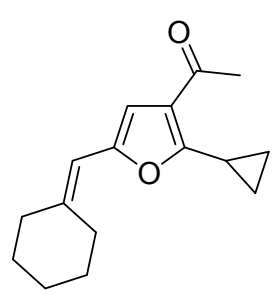
liquid (66 mg, 68\%), $\mathrm{R}_{f}=0.6(\mathrm{PE}: \mathrm{EA}=15: 1) .{ }^{1} \mathrm{H} \mathrm{NMR}(500 \mathrm{MHz}$, $\left.\mathrm{CDCl}_{3}, \mathrm{ppm}\right) \delta 6.30(\mathrm{~s}, 1 \mathrm{H}), 5,80(\mathrm{~s}, 1 \mathrm{H}), 2.80-2.75(\mathrm{~m}, 1 \mathrm{H}), 2.48$ $(\mathrm{t}, J=5.5 \mathrm{~Hz}, 2 \mathrm{H}), 2.41(\mathrm{~s}, 3 \mathrm{H}), 2.19(\mathrm{t}, J=5.5 \mathrm{~Hz}, 2 \mathrm{H}), 1.61-1.57$ $(\mathrm{m}, 6 \mathrm{H}), 1.05-1.02(\mathrm{~m}, 4 \mathrm{H}) .{ }^{13} \mathrm{C}$ NMR $\left(125.8 \mathrm{MHz}, \mathrm{CDCl}_{3}, \mathrm{ppm}\right) \delta$ $194.3,161.0,149.8,144.4,122.4,110.1,107.8,37.6,30.2,29.3$, 28.5, 27.5, 26.4, 9.6, 8.9. IR (KBr): 3443, 2928, 2854, 2361, 2335, $1672,1574,806 \mathrm{~cm}^{-1}$. HRMS (ESI) $\mathrm{m} / z$ calcd for $\left[\mathrm{C}_{16} \mathrm{H}_{20} \mathrm{O}_{2}+\mathrm{H}\right]^{+} 245.1538$, found 245.1536 .

methyl 5-(cyclohexylidenemethyl)-2-methylfuran-3-carboxylate (2r). Yellow<smiles>COC(=O)c1cc(C=C2CCCCC2)oc1C</smiles>
liquid (64 mg, 68\%), $\mathrm{R}_{f}=0.5$ (PE:EA = 15:1). ${ }^{1} \mathrm{H}$ NMR $(500$ $\left.\mathrm{MHz}, \mathrm{CDCl}_{3}, \mathrm{ppm}\right) \delta 6.38(\mathrm{~s}, 1 \mathrm{H}), 5.87(\mathrm{~s}, 1 \mathrm{H}), 3.81(\mathrm{~s}, 3 \mathrm{H}), 2.56$ $(\mathrm{s}, 3 \mathrm{H}), 2.53(\mathrm{t}, J=5.0 \mathrm{~Hz}, 2 \mathrm{H}), 2.22(\mathrm{t}, J=5.0 \mathrm{~Hz}, 2 \mathrm{H}), 1.64$ 1.57 (m, 6H). ${ }^{13} \mathrm{C}$ NMR $\left(125.8 \mathrm{MHz}, \mathrm{CDCl}_{3}, \mathrm{ppm}\right) \delta 164.7,157.3$, 151.0, 144.6, 114.2, 110.3, 107.7, 51.2, 37.5, 30.1, 28.5, 27.5, 26.4, 13.8. IR (KBr): 3444, 2925, 2361, 2335, 1645, 1540, 1515, $752 \mathrm{~cm}^{-1}$. HRMS (ESI) $\mathrm{m} / z$ calcd for $\left[\mathrm{C}_{14} \mathrm{H}_{18} \mathrm{O}_{3}+\mathrm{H}\right]^{+} 235.1329$, found 235.1329 .

5-(cyclohexylidenemethyl)-2-methyl-3-(phenylsulfonyl)furan (2s). Yellow liquid

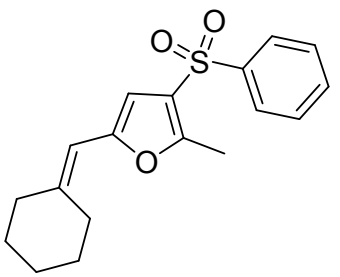


(85 mg, 70\%), $\mathrm{R}_{f}=0.6(\mathrm{PE}: \mathrm{EA}=15: 1) .{ }^{1} \mathrm{H} \mathrm{NMR}\left(500 \mathrm{MHz}, \mathrm{CDCl}_{3}, \mathrm{ppm}\right) \delta 7.9(\mathrm{~d}, J$ $=7.5 \mathrm{~Hz}, 2 \mathrm{H}), 7.54-7.46(\mathrm{~m}, 3 \mathrm{H}), 6.29(\mathrm{~s}, 1 \mathrm{H}), 5.78(\mathrm{~m}, 1 \mathrm{H}), 2.54(\mathrm{~s}, 3 \mathrm{H}), 2.45(\mathrm{t}, J=$ $5.0 \mathrm{~Hz}, 2 \mathrm{H}), 2.15(\mathrm{t}, J=5.0 \mathrm{~Hz}, 2 \mathrm{H}), 1.56-1.52(\mathrm{~m}, 6 \mathrm{H}) .{ }^{13} \mathrm{C}$ NMR $(125.8 \mathrm{MHz}$, $\left.\mathrm{CDCl}_{3}, \mathrm{ppm}\right) \delta 154.3,152.0,146.4,142.6,133.0,129.2,126.8,123.5,109.6,106.5$, 37.5, 30.2, 28.4, 27.5, 26.3, 13.0. IR (KBr): 3442, 2929, 2854, 2361, 1652, 1585, 1447, 1314, 1157, 726, $608 \mathrm{~cm}^{-1}$. HRMS (ESI) $\mathrm{m} / z$ calcd for $\left[\mathrm{C}_{18} \mathrm{H}_{20} \mathrm{O}_{3} \mathrm{~S}+\mathrm{H}\right]^{+}$ 334.1481, found 334.1471.

1-(5-(cyclopentylidenemethyl)-2-methylfuran-3-yl)ethanone (2t). Yellow liquid

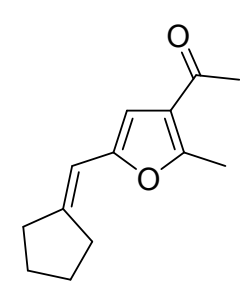

(57 mg, 70\%), $\mathrm{R}_{f}=0.6$ (PE:EA = 15:1). ${ }^{1} \mathrm{H} \mathrm{NMR} \mathrm{(500} \mathrm{MHz,} \mathrm{CDCl}_{3}$, ppm) $\delta 6.25(\mathrm{~s}, 1 \mathrm{H}), 6.10(\mathrm{t}, J=2.5 \mathrm{~Hz}, 1 \mathrm{H}), 2.53(\mathrm{~s}, 3 \mathrm{H}), 2.45(\mathrm{t}, J=$ $7 \mathrm{~Hz}, 2 \mathrm{H}), 2.41(\mathrm{t}, J=7 \mathrm{~Hz}, 2 \mathrm{H}), 2.36(\mathrm{~s}, 3 \mathrm{H}), 1.79-1.3(\mathrm{~m}, 2 \mathrm{H})$, 1.67-1.61 (m, 2H). ${ }^{13} \mathrm{C}$ NMR (125.8 $\left.\mathrm{MHz}, \mathrm{CDCl}_{3}, \mathrm{ppm}\right) \delta 194.3$, $156.3,151.9,147.6,122.8,109.0,106.1,35.3,31.7,29.1,26.8,26.2$, 14.4. IR (KBr): 3442, 2956, 2871, 2361, 1675, 1581, $945 \mathrm{~cm}^{-1}$. HRMS (ESI) $m / z$ calcd for $\left[\mathrm{C}_{13} \mathrm{H}_{16} \mathrm{O}_{2}+\mathrm{H}\right]^{+} 205.1222$, found 205.1223.

(5-(cyclopentylidenemethyl)-2-methylfuran-3-yl)(phenyl)methanone (2u). Yellow

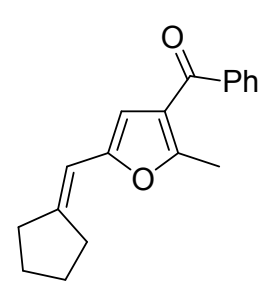
liquid (66 mg, 62\%), $\mathrm{R}_{f}=0.5$ (PE:EA = 15:1). ${ }^{1} \mathrm{H}$ NMR $(500 \mathrm{MHz}$, $\left.\mathrm{CDCl}_{3}, \mathrm{ppm}\right) \delta$ 7.95-7.93 (m, 2H), 7.45-7.38 (m, 3H), $6.47(\mathrm{~s}, 1 \mathrm{H})$, $6.24(\mathrm{t}, J=2.0 \mathrm{~Hz}, 1 \mathrm{H}), 2.62(\mathrm{t}, J=7.0 \mathrm{~Hz}, 2 \mathrm{H}), 2.49(\mathrm{t}, J=7.0 \mathrm{~Hz}$, $2 \mathrm{H}), 2.42$ (s, 3H), 1.86-1.80 (m, 2H), 1.73-1.69 (m, 2H). ${ }^{13} \mathrm{C} \mathrm{NMR}$ $\left(125.8 \mathrm{MHz}, \mathrm{CDCl}_{3}, \mathrm{ppm}\right) \delta 194.4,154.2,152.9,149.1,130.2$, $129.3,128.3,128.2,123.7,108.9,108.5,35.6,31.9,29.9,26.9$, 26.3. IR (KBr): 3443, 2956, 2870, 2361, 1679, 1525, 1483, 938, $697 \mathrm{~cm}^{-1}$. HRMS (ESI) $m / z$ calcd for $\left[\mathrm{C}_{18} \mathrm{H}_{18} \mathrm{O}_{2}+\mathrm{H}\right]^{+} 267.1380$, found 267.1380 .

5-(cyclopentylidenemethyl)-2-methyl-3-(phenylsulfonyl)furan (2v). Yellow liquid

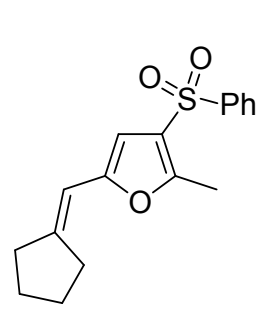
(75 mg, 62\%), $\mathrm{R}_{f}=0.6$ (PE:EA = 15:1). ${ }^{1} \mathrm{H}$ NMR $(500 \mathrm{MHz}$, $\left.\mathrm{CDCl}_{3}, \mathrm{ppm}\right) \delta 7.91(\mathrm{t}, J=7.0 \mathrm{~Hz}, 2 \mathrm{H}), 7.56(\mathrm{t}, J=7.0 \mathrm{~Hz}, 1 \mathrm{H})$, $7.50(\mathrm{t}, J=7.0 \mathrm{~Hz}, 2 \mathrm{H}), 6.25(\mathrm{~s}, 1 \mathrm{H}), 6.08(\mathrm{t}, J=2.5 \mathrm{~Hz}, 1 \mathrm{H}), 2.55$ (s, 3H), 2.41 (q, $J=4.5 \mathrm{~Hz}, 7.0 \mathrm{~Hz}, 4 \mathrm{H}), 1.79-1.73(\mathrm{~m}, 2 \mathrm{H}), 1.67-$ $1.61(\mathrm{~m}, 2 \mathrm{H}) .{ }^{13} \mathrm{C}$ NMR $\left(125.8 \mathrm{MHz}, \mathrm{CDCl}_{3}, \mathrm{ppm}\right) \delta 154.1,152.9$, $149.5,142.7,133.0,129.2,126.8,123.8,108.5,105.1,35.4,31.8$, 26.8, 26.2, 13.0. IR (KBr): 3443, 2955, 2870, 2361, 1652, 1585, 1549, 1314, 1156, $725,607 \mathrm{~cm}^{-1}$. HRMS (ESI) $\mathrm{m} / z$ calcd for $\left[\mathrm{C}_{17} \mathrm{H}_{18} \mathrm{O}_{3} \mathrm{~S}+\mathrm{H}\right]^{+} 303.1046$, found 303.1049 .

1-(5-(cyclopropyldimethoxymethyl)-2-methylfuran-3-yl)ethan-1-one (3a). Yellow

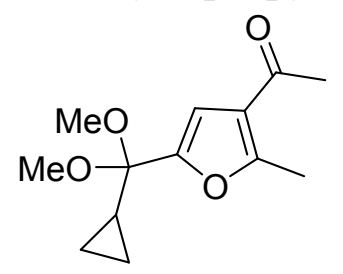
liquid (34 mg, 72\%), $\mathrm{R}_{f}=0.6$ (PE:EA = 8:1). ${ }^{1} \mathrm{H}$ NMR (500 $\left.\mathrm{MHz}, \mathrm{CDCl}_{3}, \mathrm{ppm}\right) \delta 6.60(\mathrm{~s}, 1 \mathrm{H}), 3.23(\mathrm{~s}, 6 \mathrm{H}), 2.58(\mathrm{~s}, 3 \mathrm{H})$, $2.39(\mathrm{~s}, 3 \mathrm{H}), 1.15-1.01(\mathrm{~m}, 1 \mathrm{H}), 0.68-0.57(\mathrm{~m}, 2 \mathrm{H}), 0.56-0.43$ (m, 2H). ${ }^{13} \mathrm{C}$ NMR $\left(125.8 \mathrm{MHz}, \mathrm{CDCl}_{3}, \mathrm{ppm}\right) \delta 194.3,158.0$, 151.7, 121.6, 109.2, 99.0, 49.4, 29.1, 16.7, 14.5, 1.6. IR (KBr): 
3420, 2973, 2927, 2361, 2335, 1678, 1091, 1050, $881 \mathrm{~cm}^{-1}$. HRMS (ESI) $\mathrm{m} / z$ calcd for $\left[\mathrm{C}_{13} \mathrm{H}_{18} \mathrm{O}_{4}+\mathrm{H}\right]^{+} 239.1274$, found 239.1278.

(5-(cyclopropyldimethoxymethyl)-2-methylfuran-3-yl)(phenyl)methanone (3b).

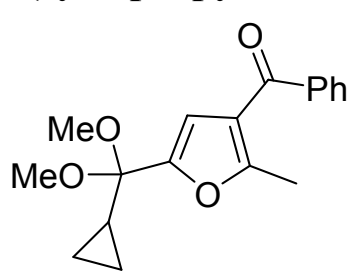

Yellow liquid (39 mg, 65\%), $\mathrm{R}_{f}=0.5$ (PE:EA = 8:1). $1 \mathrm{H}$ NMR $\left(500 \mathrm{MHz}, \mathrm{CDCl}_{3}\right) \delta$ 7.95-7.41 (m, 5H), $6.80(\mathrm{~s}, 1 \mathrm{H})$, $3.28(\mathrm{~s}, 6 \mathrm{H}), 2.44(\mathrm{~m}, 3 \mathrm{H}), 1.15-1.09(\mathrm{~m}, 1 \mathrm{H}), 0.76-0.73(\mathrm{~m}$, 2H), 0.56-0.52 (m, 2H). ${ }^{13} \mathrm{C}$ NMR (125.8 MHz, $\left.\mathrm{CDCl}_{3}, \mathrm{ppm}\right)$ $\delta 194.1,155.7,153.1,129.9,129.6,128.4,128.2,122.3$, 111.3, 98.9, 49.5, 29.9, 16.8, 1.6. IR (KBr): 3441, 2966, 2923, 2361, 2335, 1653, 1561, $939 \mathrm{~cm}^{-1}$. HRMS (ESI) $\mathrm{m} / z$ calcd for $\left[\mathrm{C}_{18} \mathrm{H}_{20} \mathrm{O}_{4}+\mathrm{H}\right]^{+}$ 301.1434 , found 301.1434 .

(5-(cyclopropyldimethoxymethyl)-2-phenylfuran-3-yl)(phenyl)methanone (3c). Yellow liquid (43 mg, 60\%), $\mathrm{R}_{f}=0.45$ (PE:EA $\left.=8: 1\right) .{ }^{1} \mathrm{H}$<smiles>COC(OC)(c1cc(C(=O)c2ccccc2)c(-c2ccccc2)o1)C1CC1</smiles>
NMR (500 MHz, $\left.\mathrm{CDCl}_{3}, \mathrm{ppm}\right) \delta 7.87-7.30(\mathrm{~m}, 10 \mathrm{H}), 6.70(\mathrm{~s}$, $1 \mathrm{H}), 3.29(\mathrm{~s}, 6 \mathrm{H}), 1.20-1.14(\mathrm{~m}, 1 \mathrm{H}), 0.81-0.78(\mathrm{~m}, 2 \mathrm{H})$, 0.59-0.55 (m, 2H). ${ }^{13} \mathrm{C}$ NMR (125.8 $\left.\mathrm{MHz}, \mathrm{CDCl}_{3}, \mathrm{ppm}\right) \delta$ $191.8, / 155.3,152.9,138.1,132.8,129.8,129.7,129.0,128.4$, $128.3,127.4,121.1,112.6,98.9,49.5,16.9,1.7$. IR (KBr): 3442, 2935, 2361, 2335, 1654, 1541, 1488, 1453, 1234, 1144, 1099, 1070, 934, 891, $728,691 \mathrm{~cm}^{-1}$. HRMS (ESI) $\mathrm{m} / z$ calcd for $\left[\mathrm{C}_{23} \mathrm{H}_{22} \mathrm{O}_{4}+\mathrm{H}\right]^{+} 363.1591$, found 363.1591 . 


\section{${ }^{1} \mathrm{H}$ and ${ }^{13} \mathrm{C}$ NMR spectra of 2a-2n, 3a-3o.}

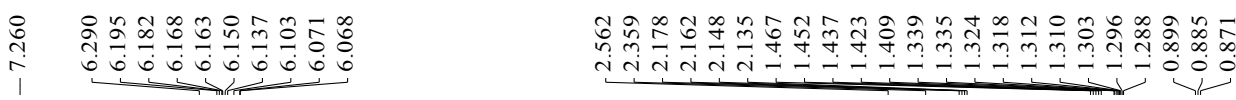
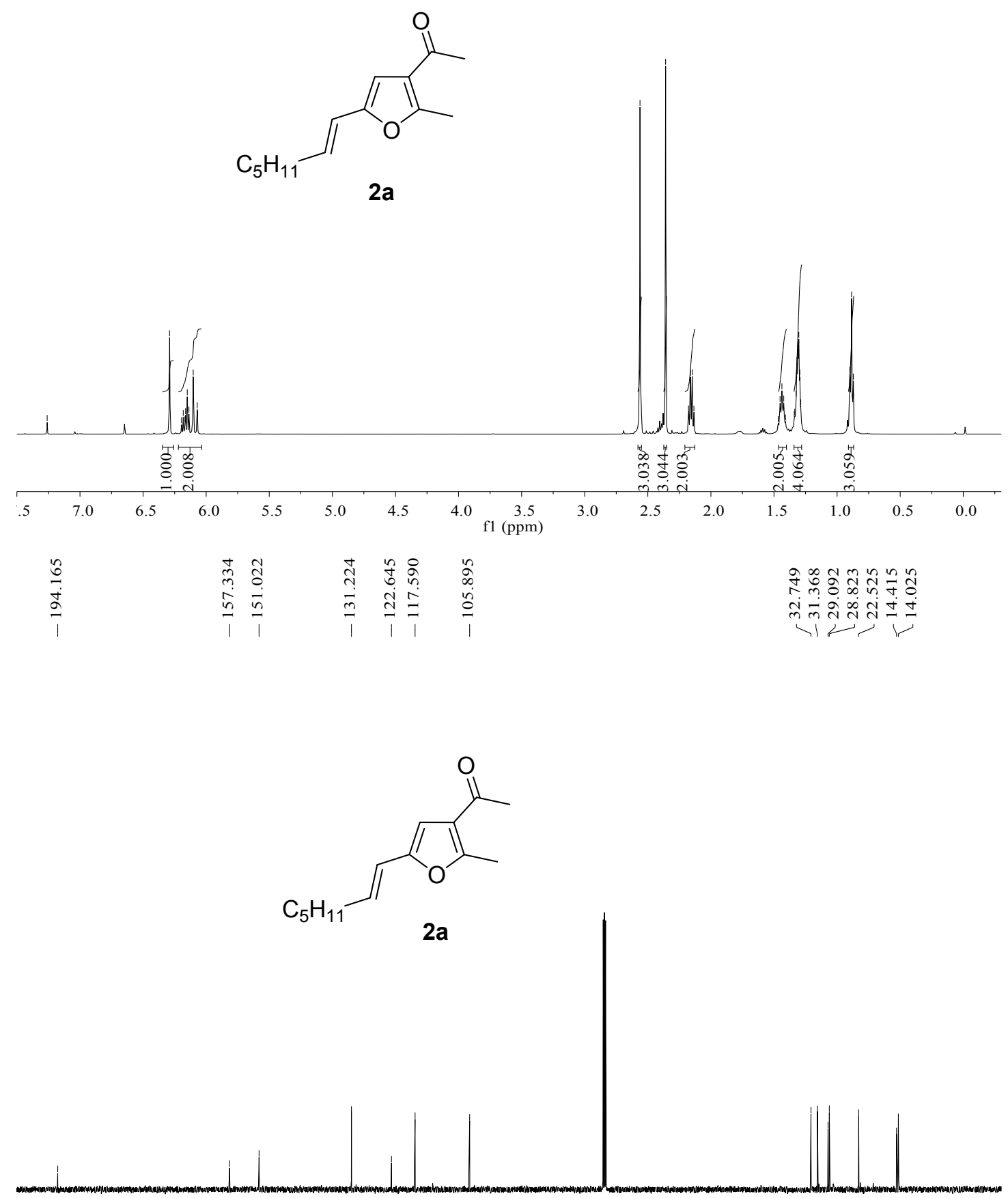

$\begin{array}{lllllllllllllllllllllllllll}200 & 190 & 180 & 170 & 160 & 150 & 140 & 130 & 120 & 110 & 100 & 90 & 80 & 70 & 60 & 50 & 40 & 30 & 20 & 10 & 0\end{array}$ 

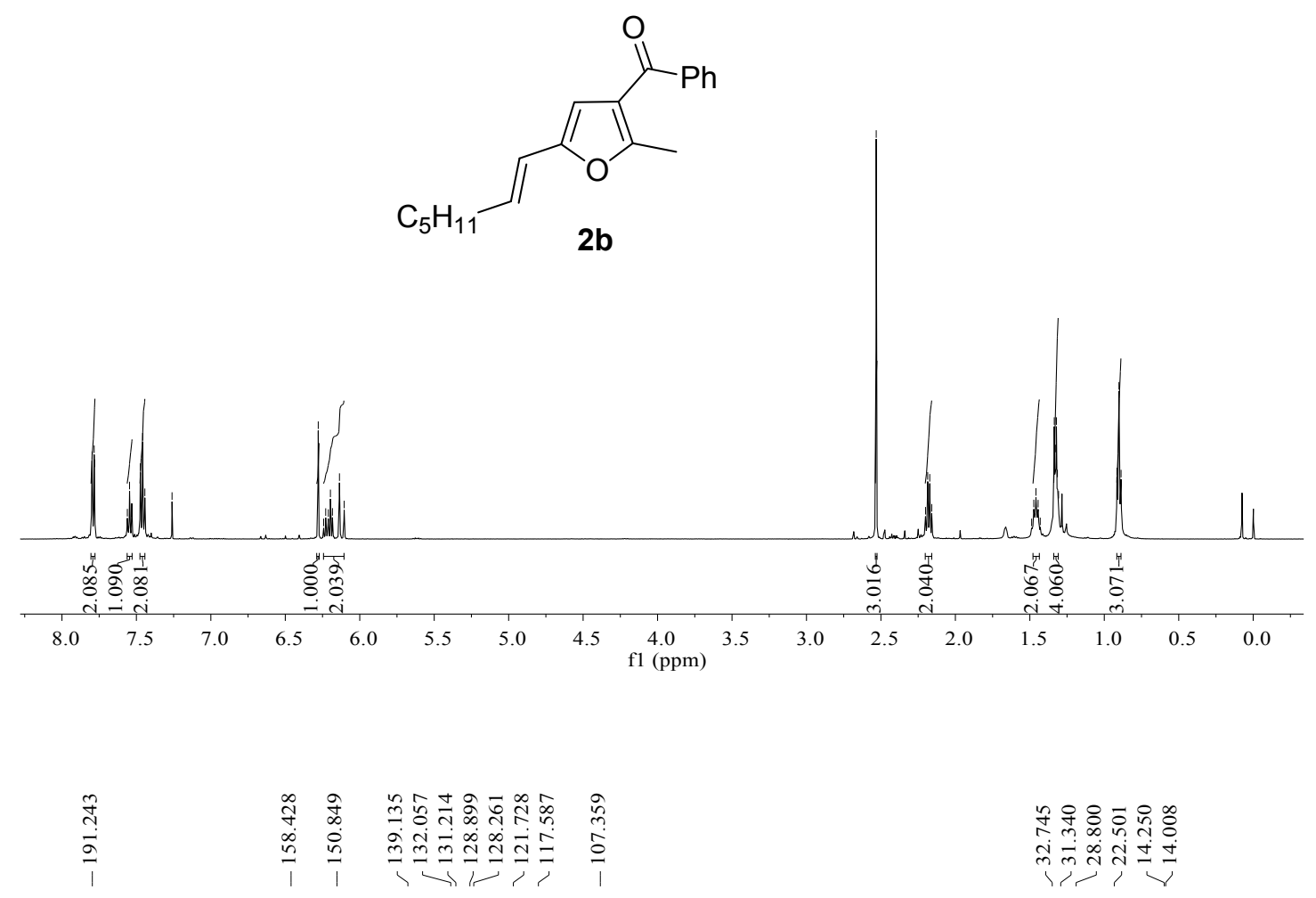

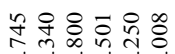

inं

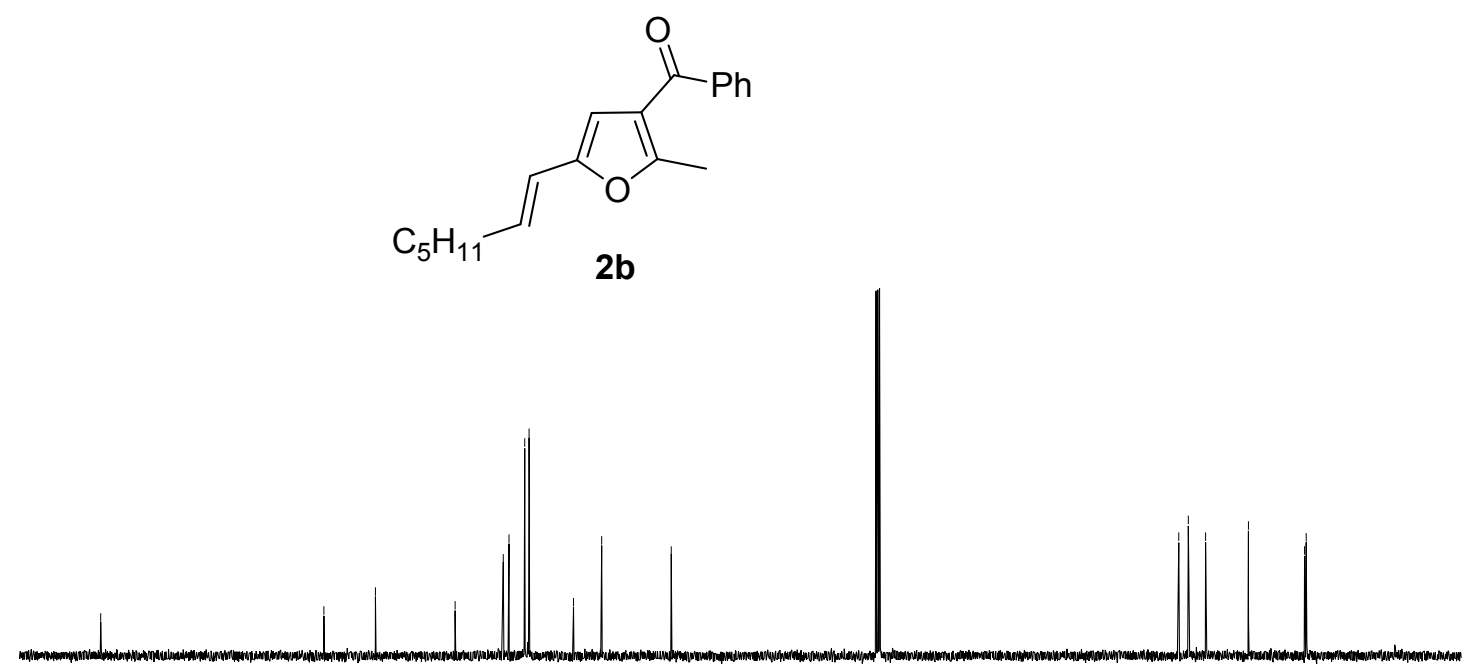

$\begin{array}{lllllllllllllllllllll}200 & 190 & 180 & 170 & 160 & 150 & 140 & 130 & 120 & 110 & 100 & 90 & 80 & 70 & 60 & 50 & 40 & 30 & 20 & 10 & 0\end{array}$ 


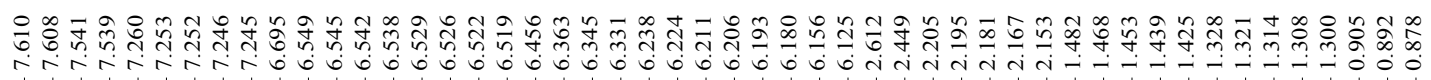

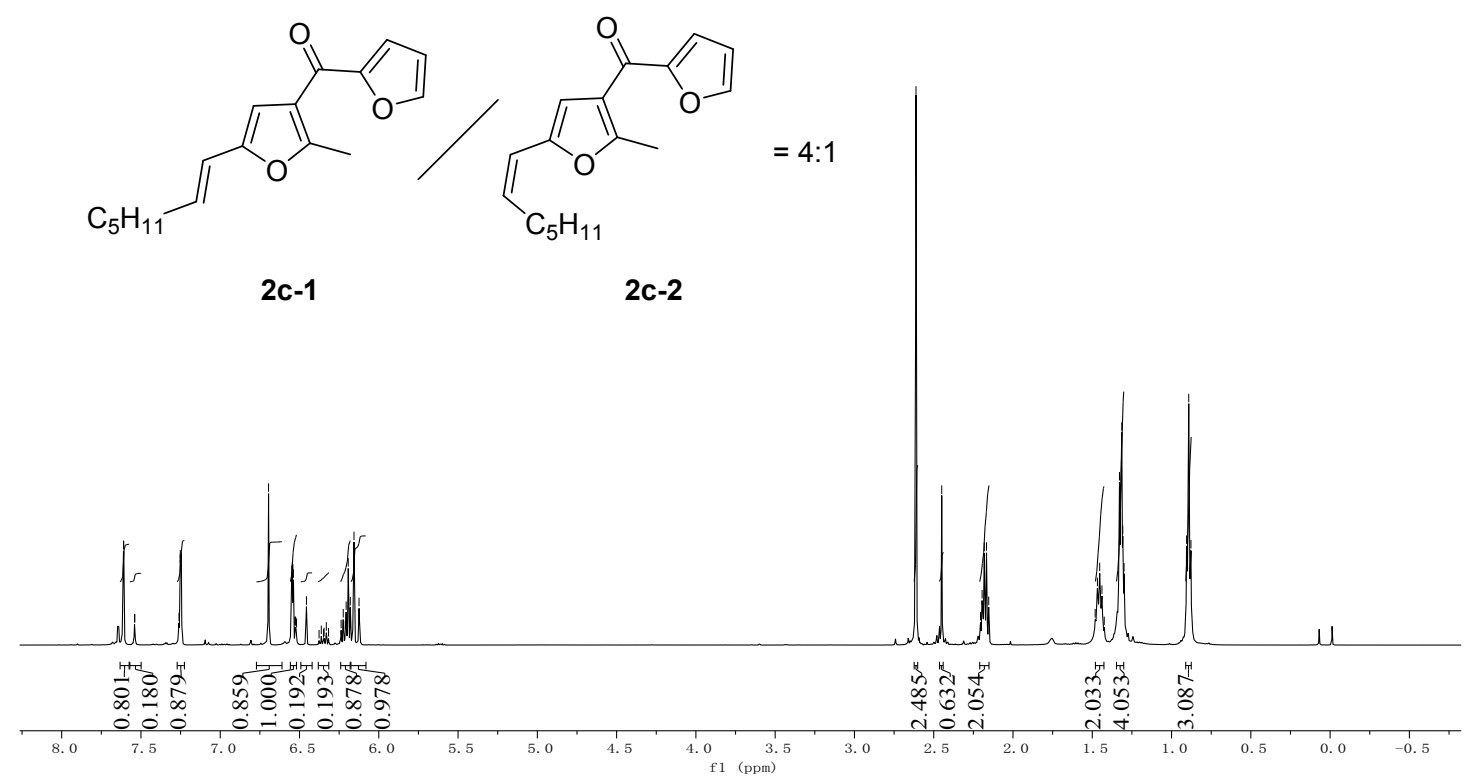

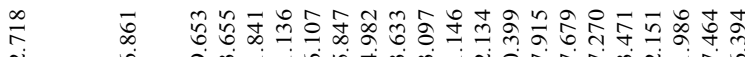

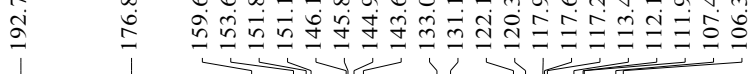

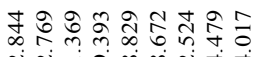

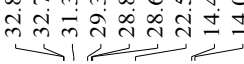

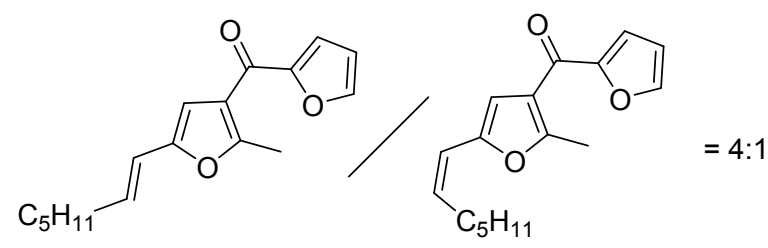

$2 c-1$

2c-2

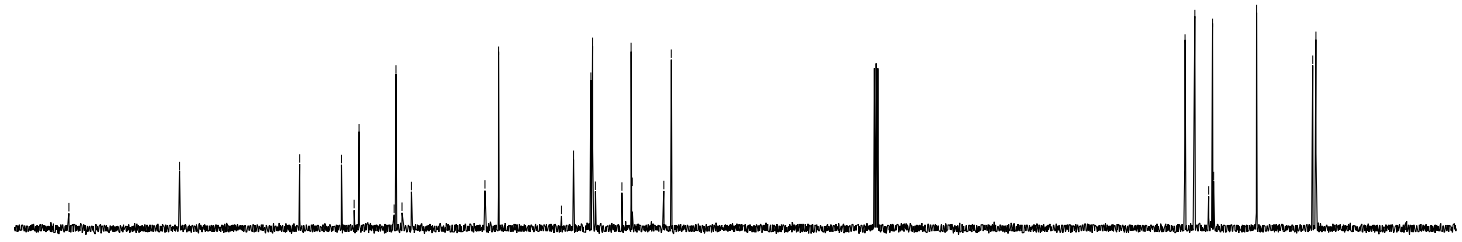




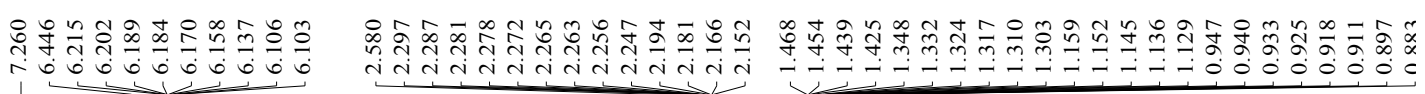
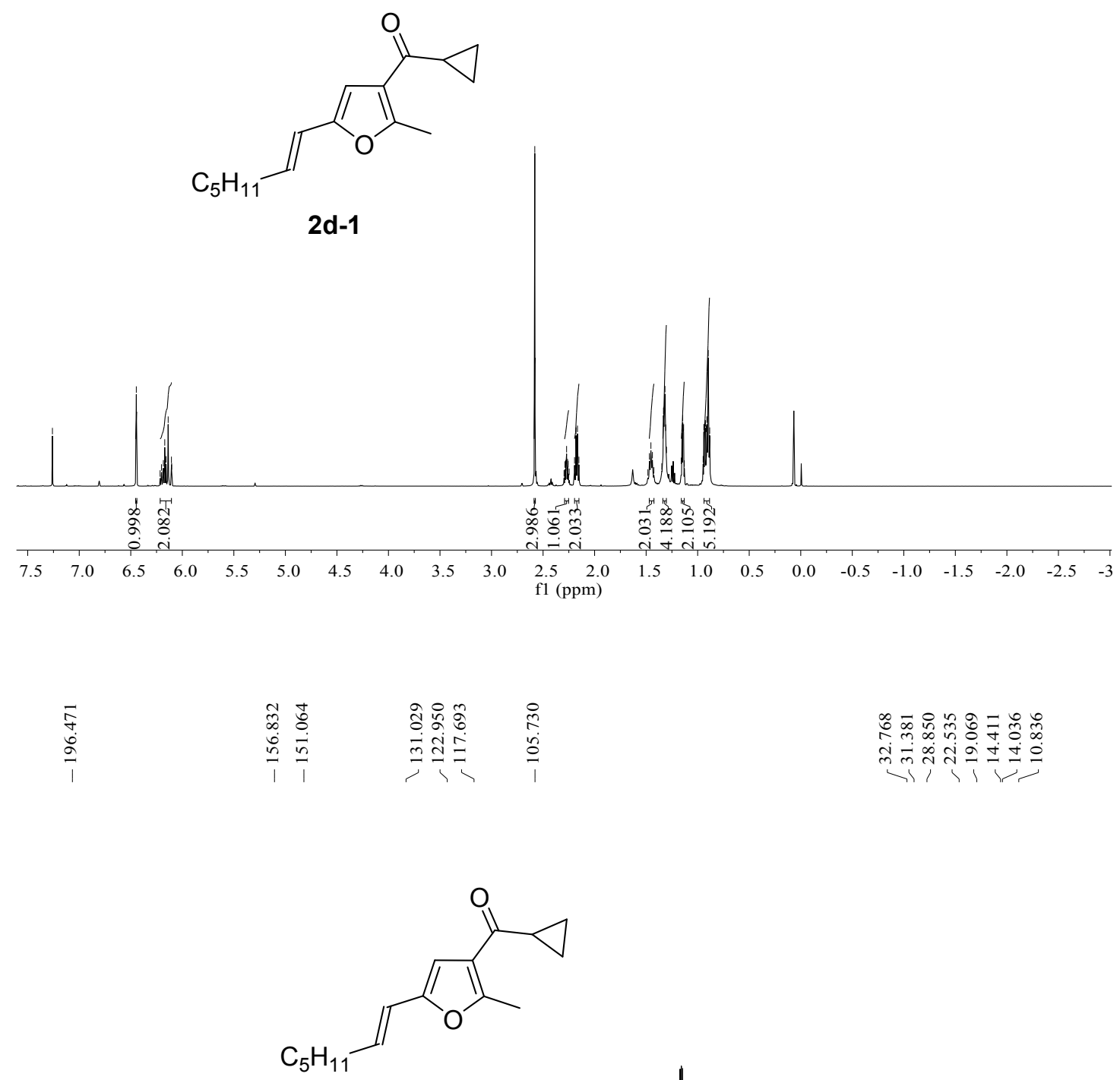

2d-1 


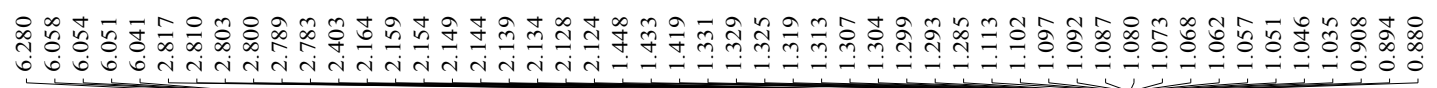

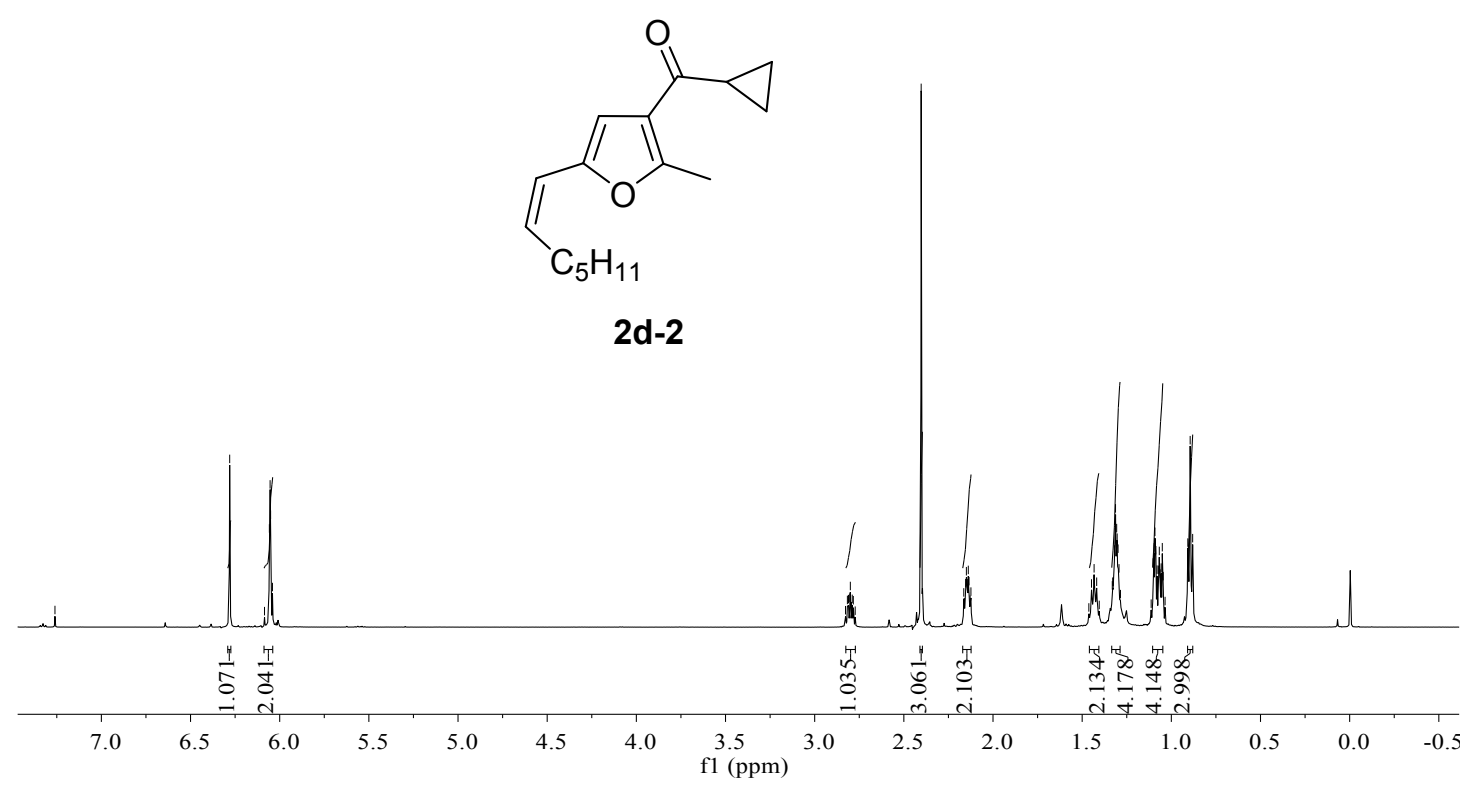

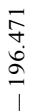

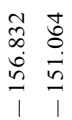

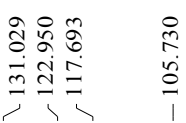

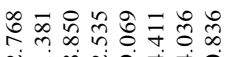
실

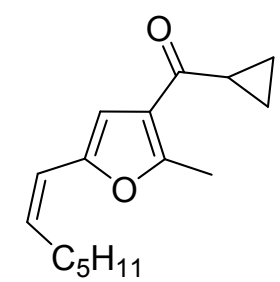

2d-2 

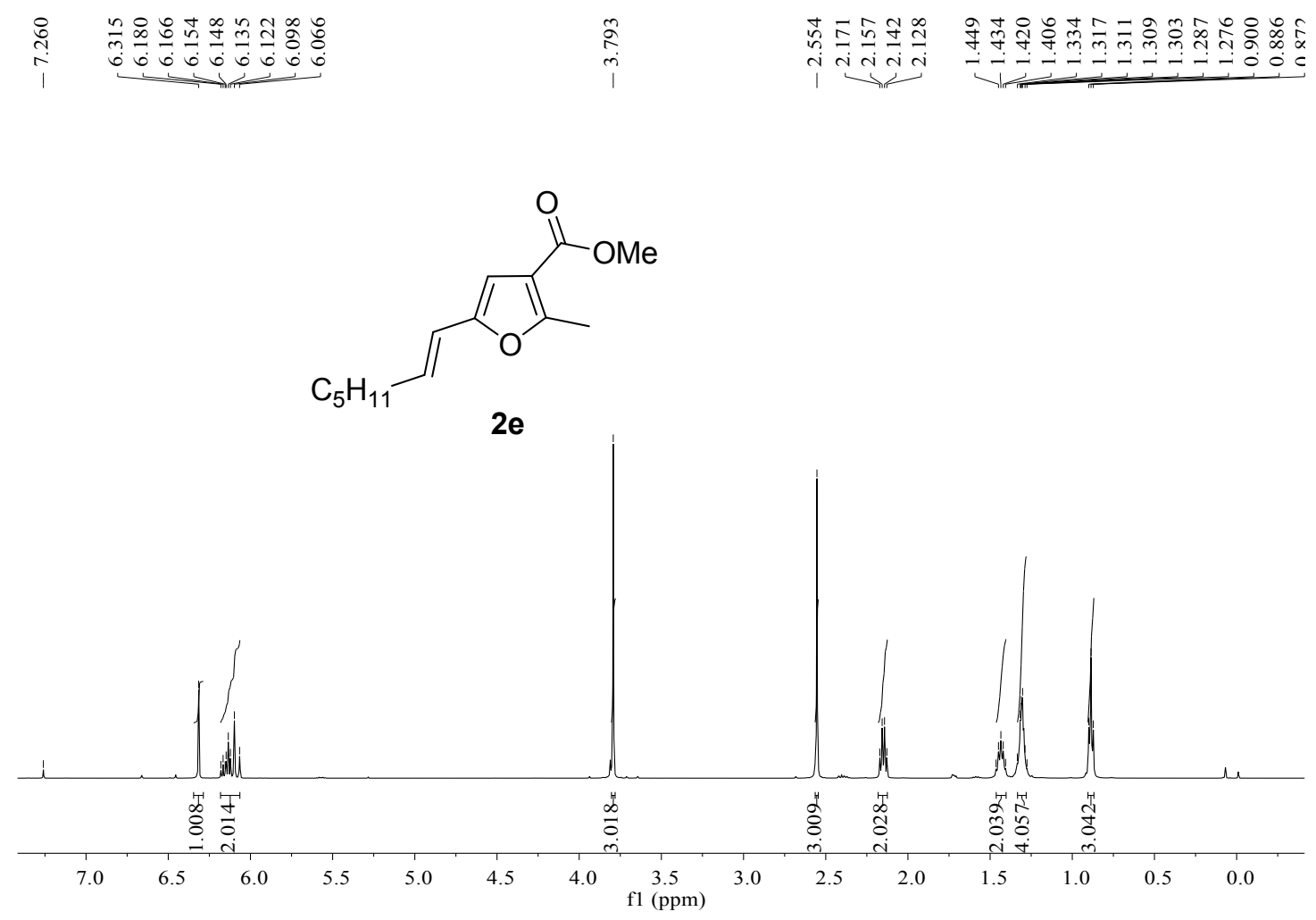

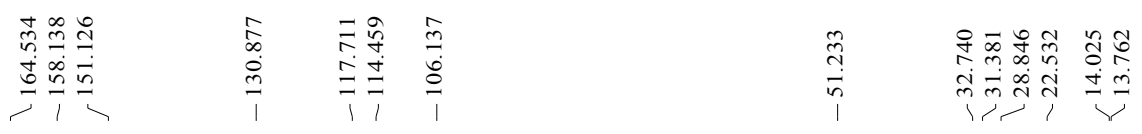
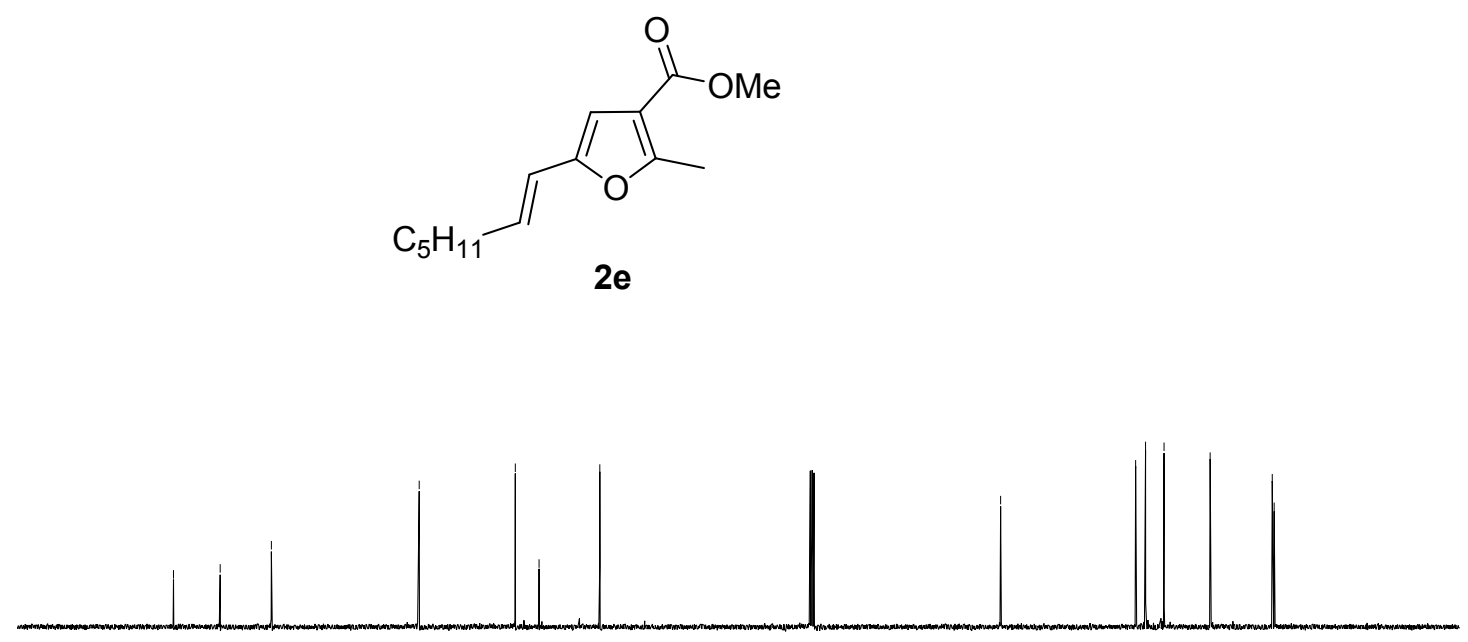

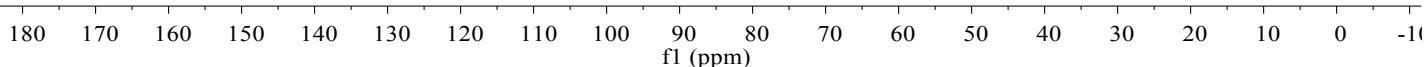


<smiles>CCCCCCCCCCCCC=Cc1cc(C(=O)OC)c(C)o1</smiles>

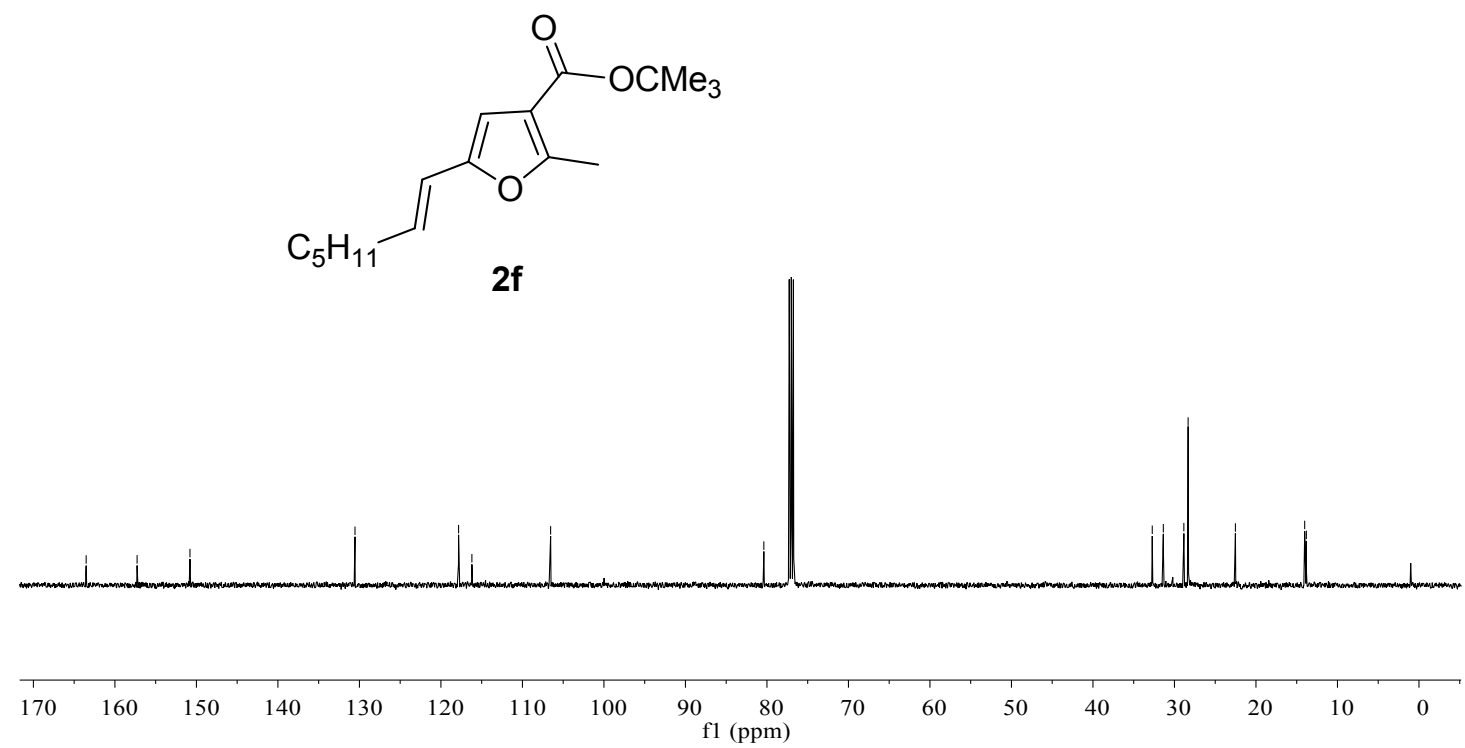


<smiles>C=CCOC(=O)c1cc(/C=C/[SbH2])oc1C</smiles><smiles>C=CCOC(=O)c1cc(/C=C/[SbH2])oc1C</smiles>

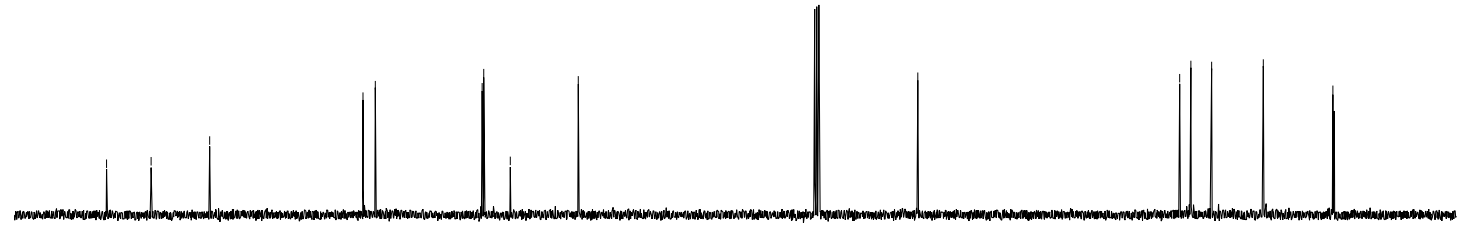

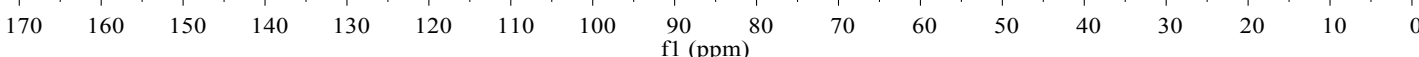




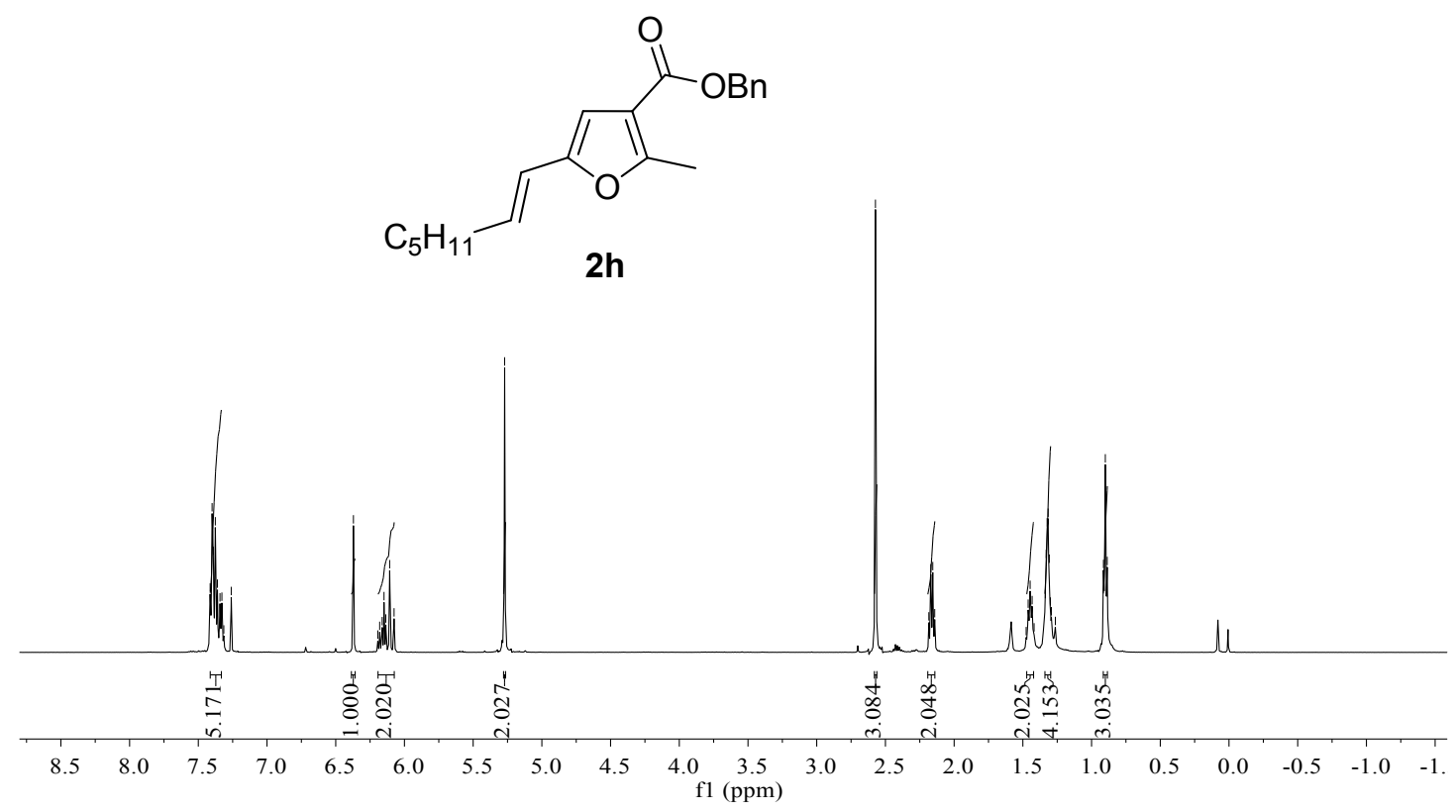<smiles>[CH2]/C=C/c1cc(C(=O)OCc2ccccc2)c(C)o1</smiles>

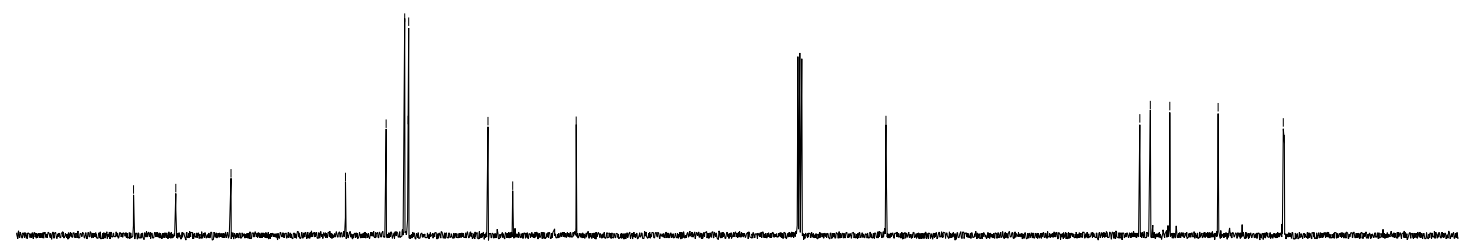


<smiles>[CH2]/C=C/c1cc(C(=O)Nc2ccccc2)c(C)o1</smiles>
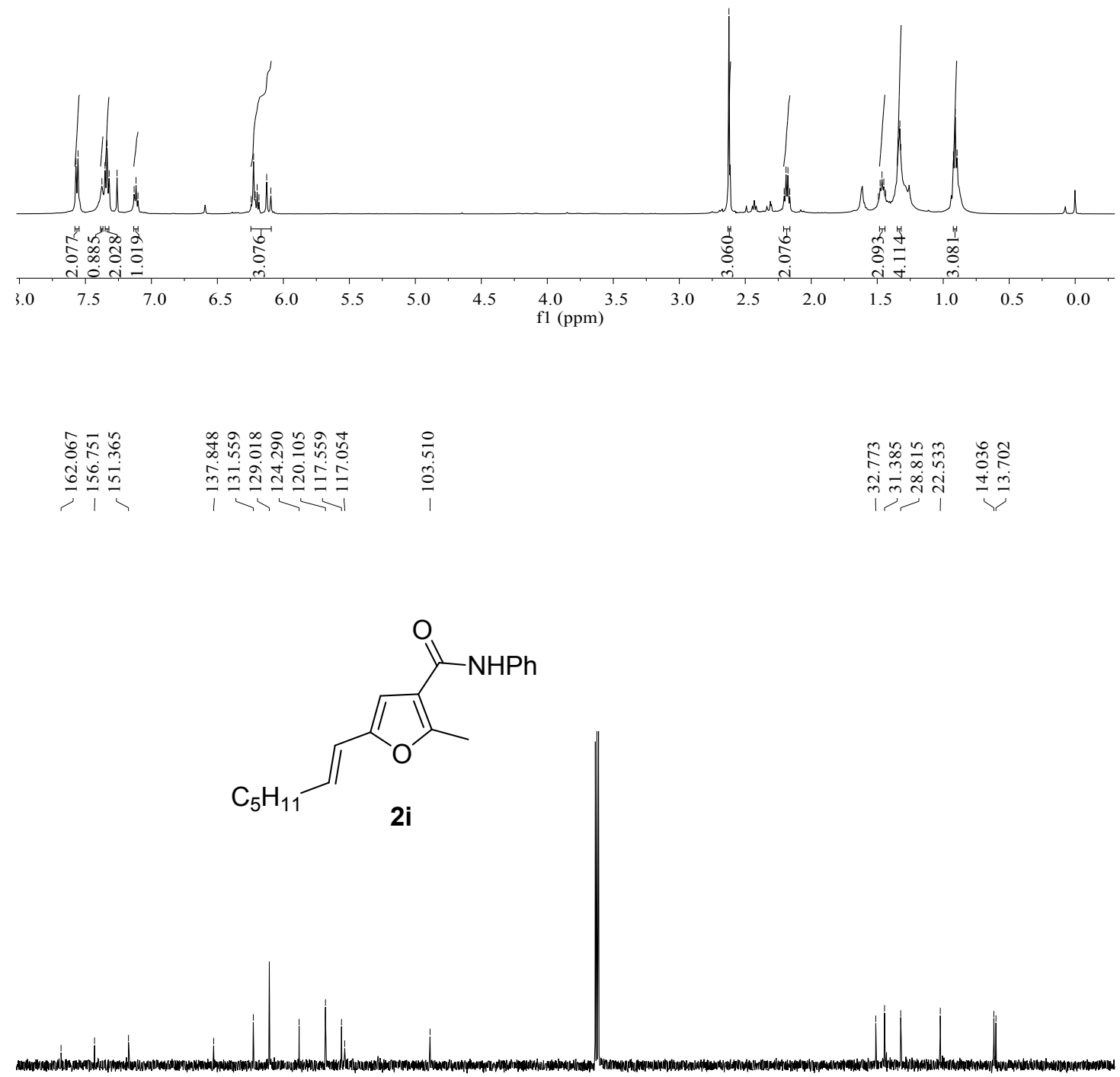

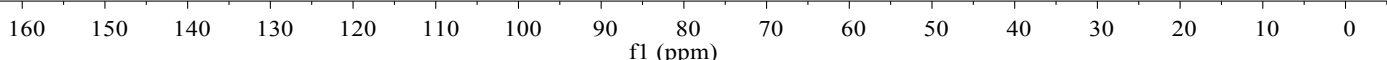



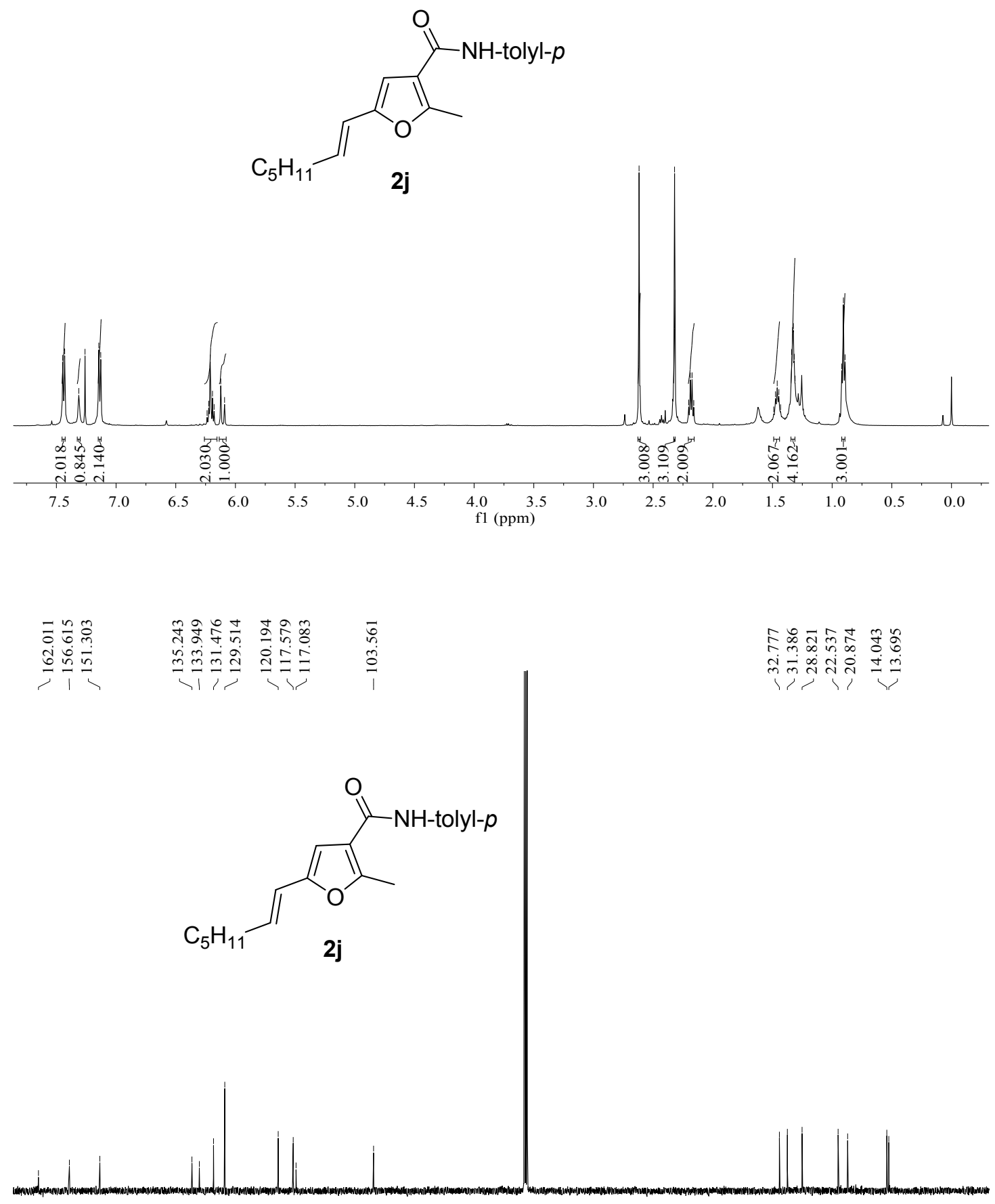

$\begin{array}{lllllllll}160 & 150 & 140 & 130 & 120 & 110 & 100 & 90 & \begin{array}{c}80 \\ \mathrm{f} 1(\mathrm{ppm})\end{array}\end{array}$ 


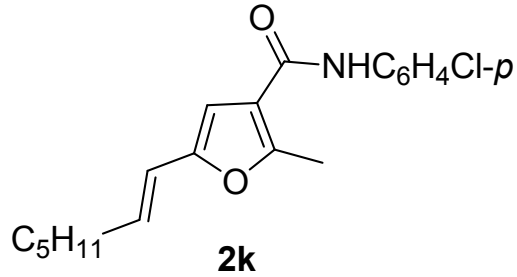

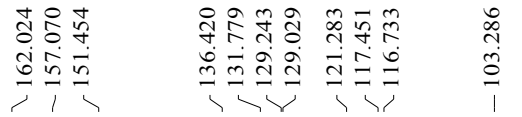<smiles>Cc1oc(/C=C/c2ccccc2)cc1C(=O)Nc1ccccc1</smiles>

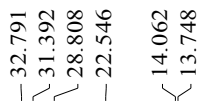

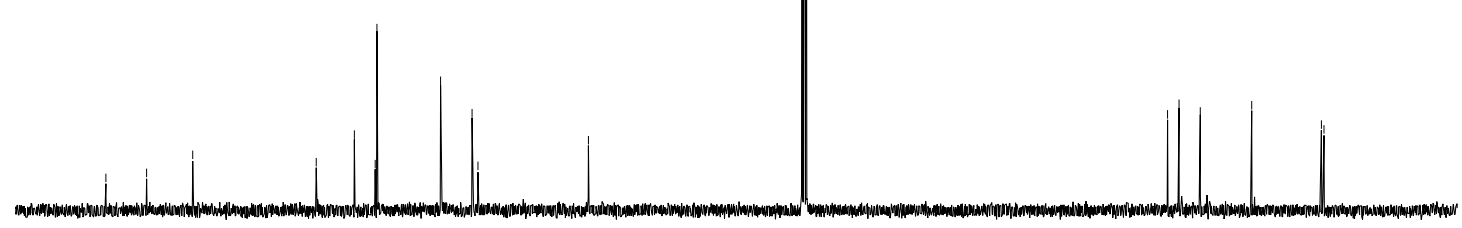

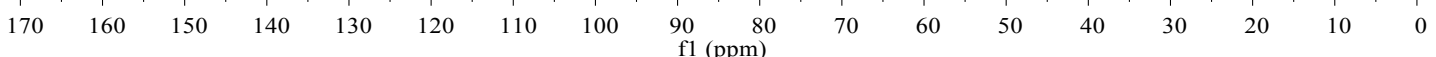


<smiles>Cc1oc(/C=C\CS)cc1S(=O)(=O)c1ccccc1S(=O)(=O)c1cc(/C=C/CSc2ccccc2)oc1C</smiles>

2I-1

21-2
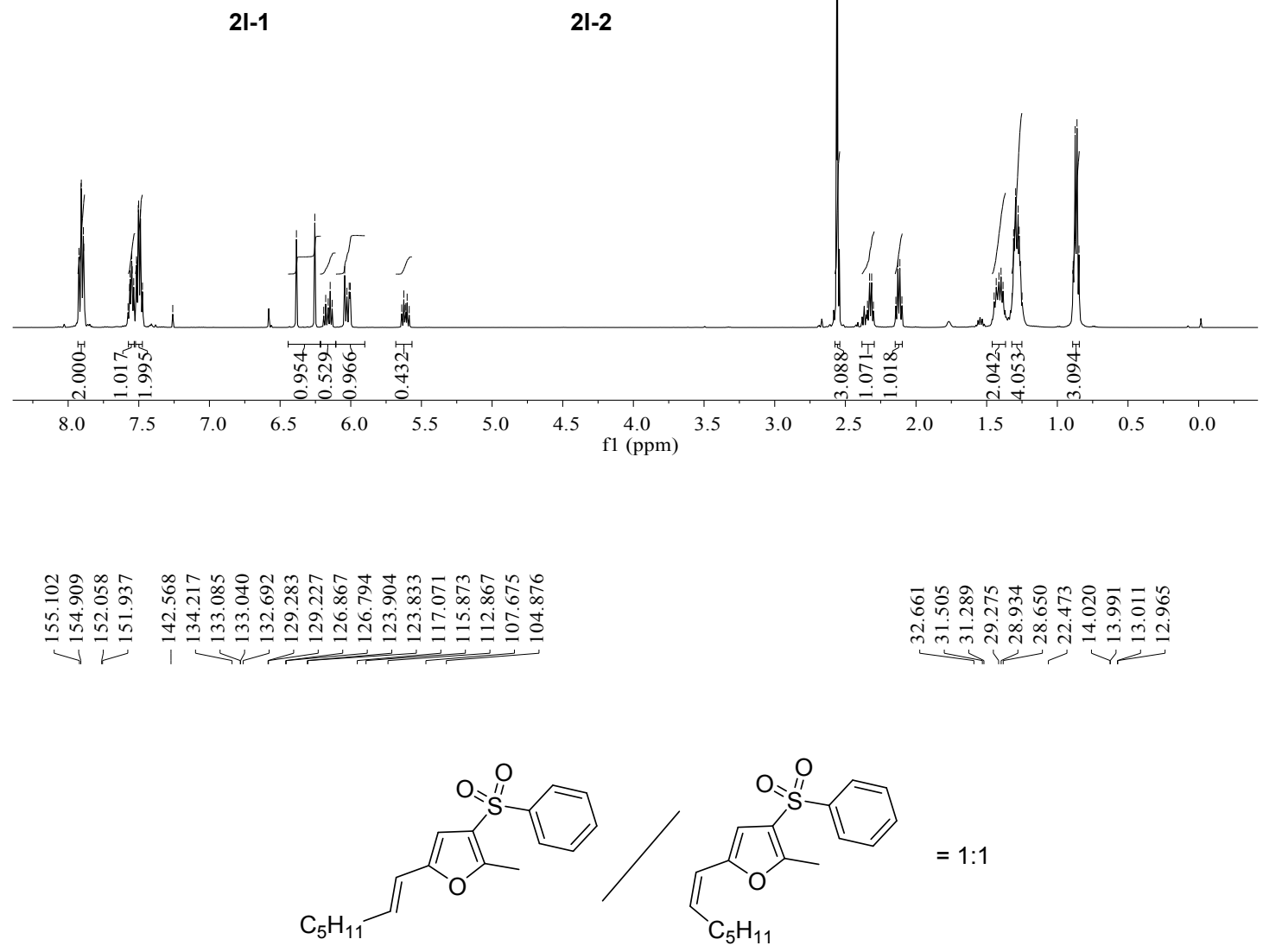

2|-1

2I-2

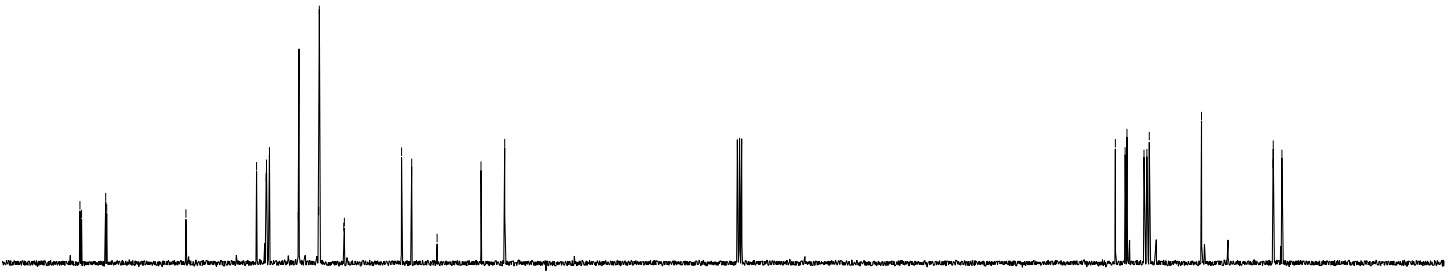

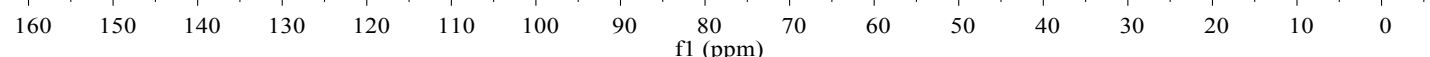




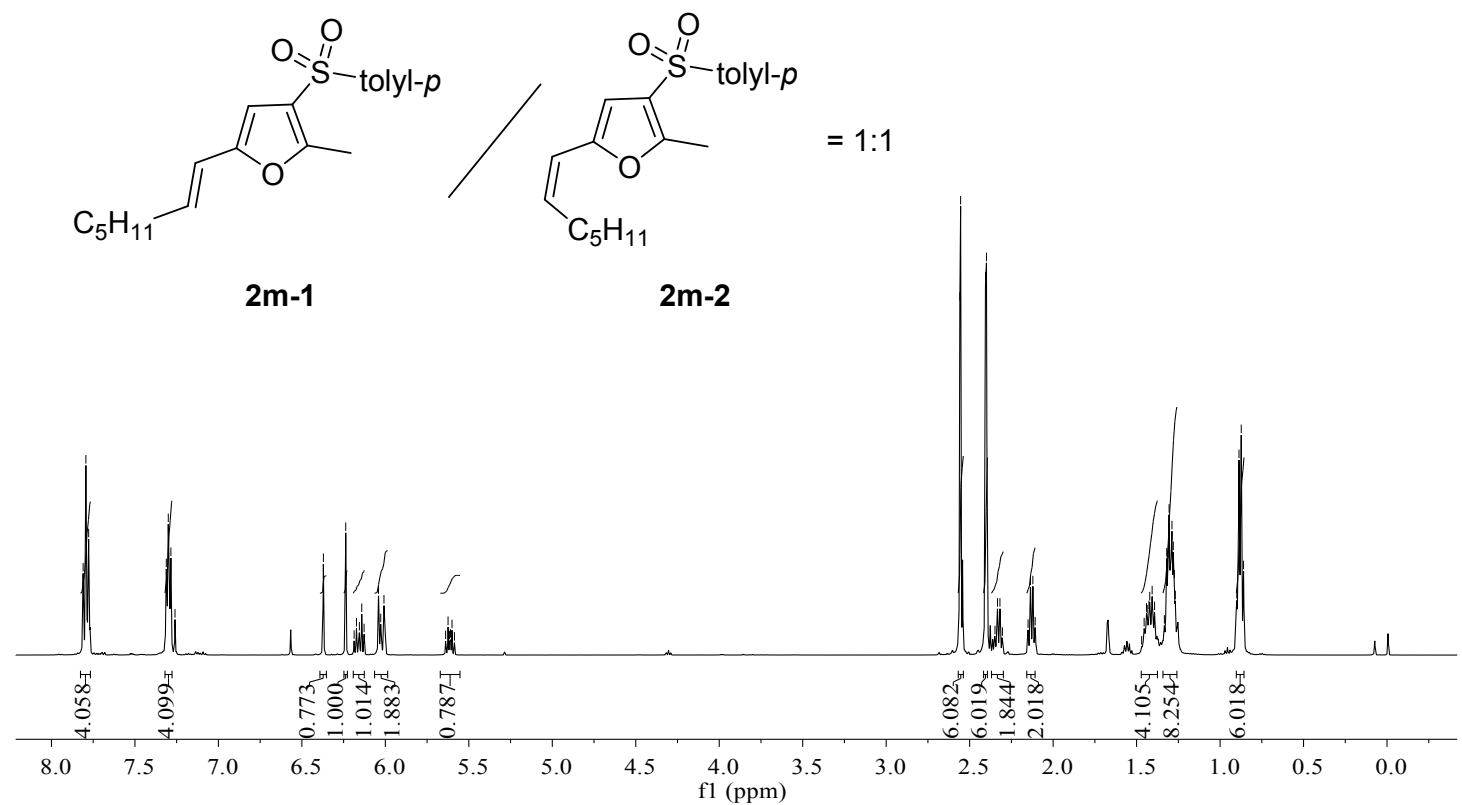<smiles>Cc1oc(/C=C/[SbH2])cc1S(=O)(=O)O[Na]</smiles>

$2 m-1$

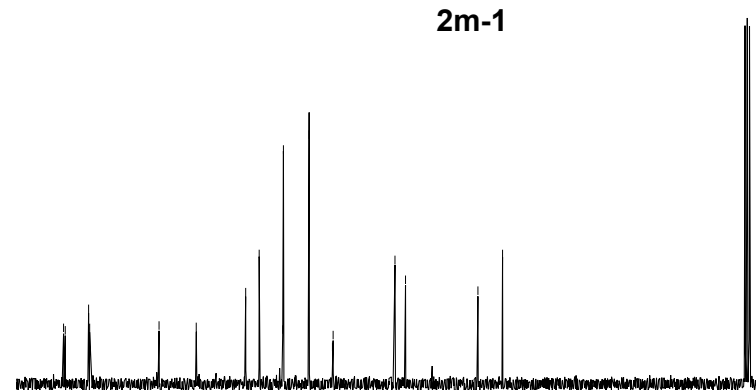<smiles>CCCCCC/C=C\c1cc(S(=O)(=O)O[Na])c(C)o1</smiles>

$2 m-2$

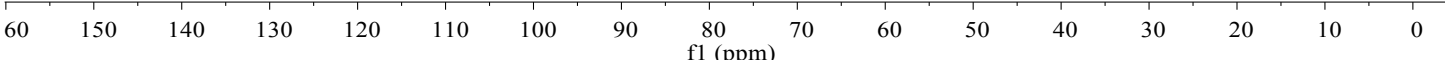


<smiles></smiles>
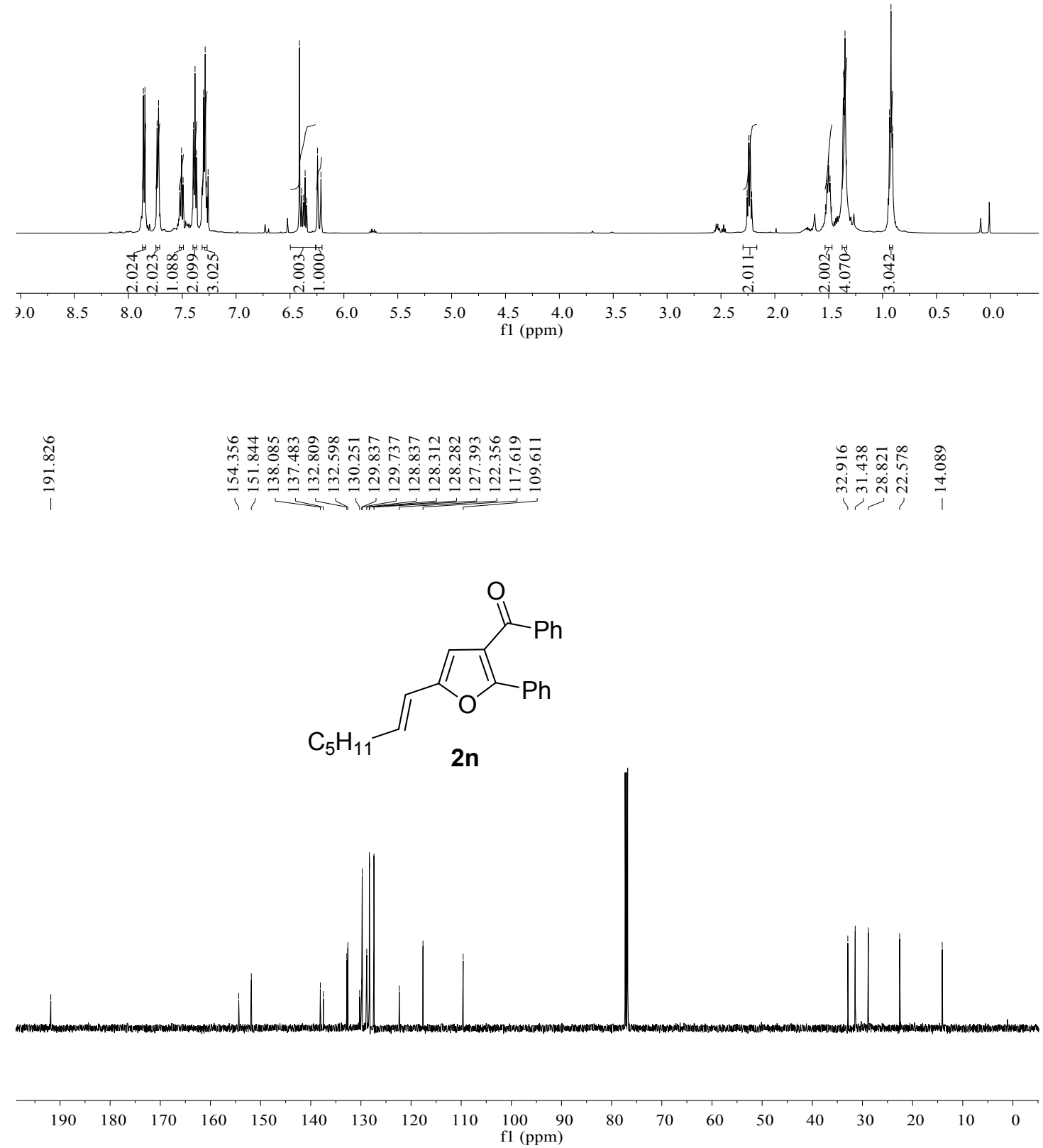


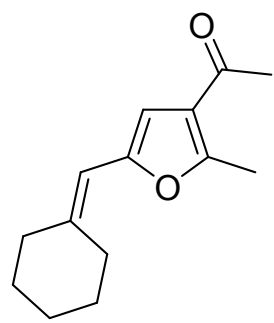

20

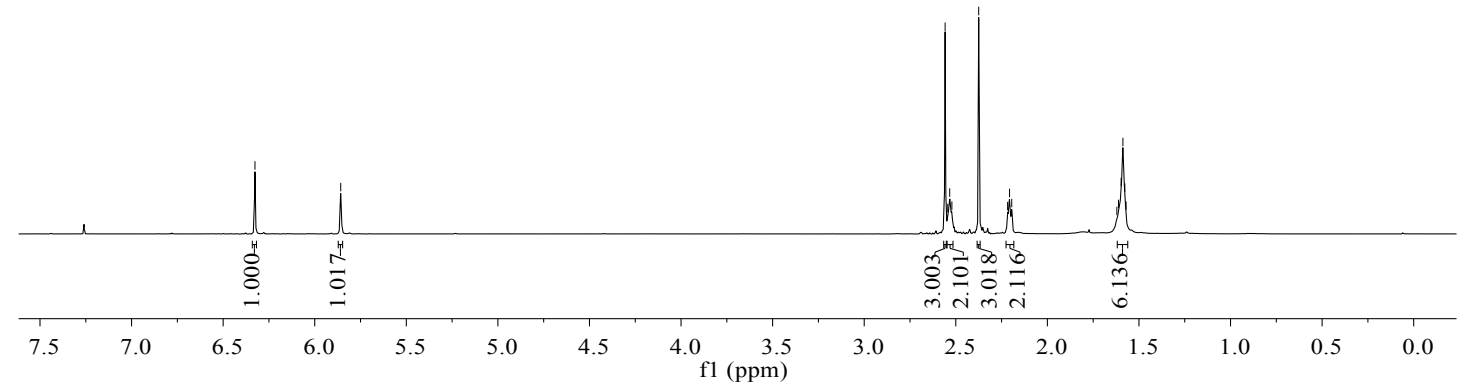

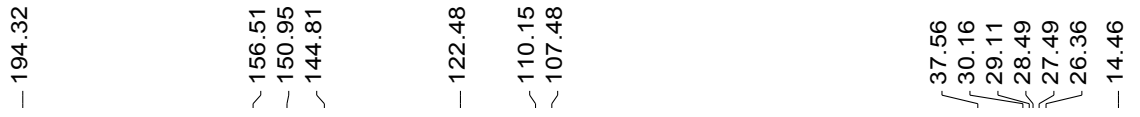

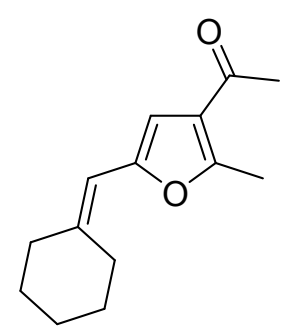

20

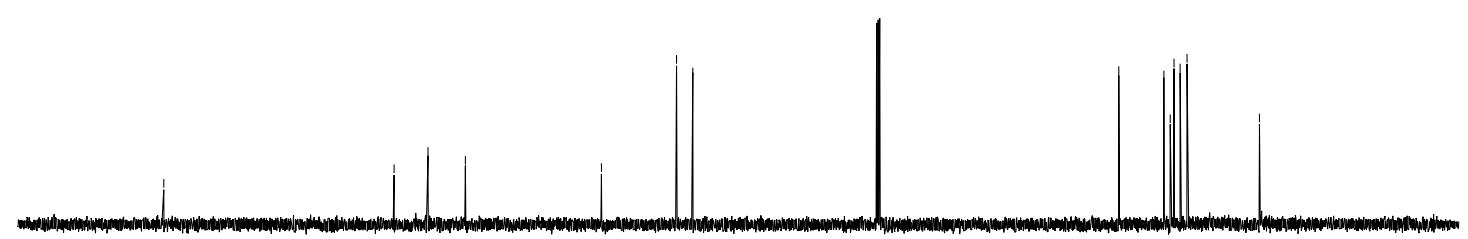

$\begin{array}{lllllllllllllllllllllll}210 & 200 & 190 & 180 & 170 & 160 & 150 & 140 & 130 & 120 & 110 & 100 & 90 & 80 & 70 & 60 & 50 & 40 & 30 & 20 & 10 & 0 & -10\end{array}$ 

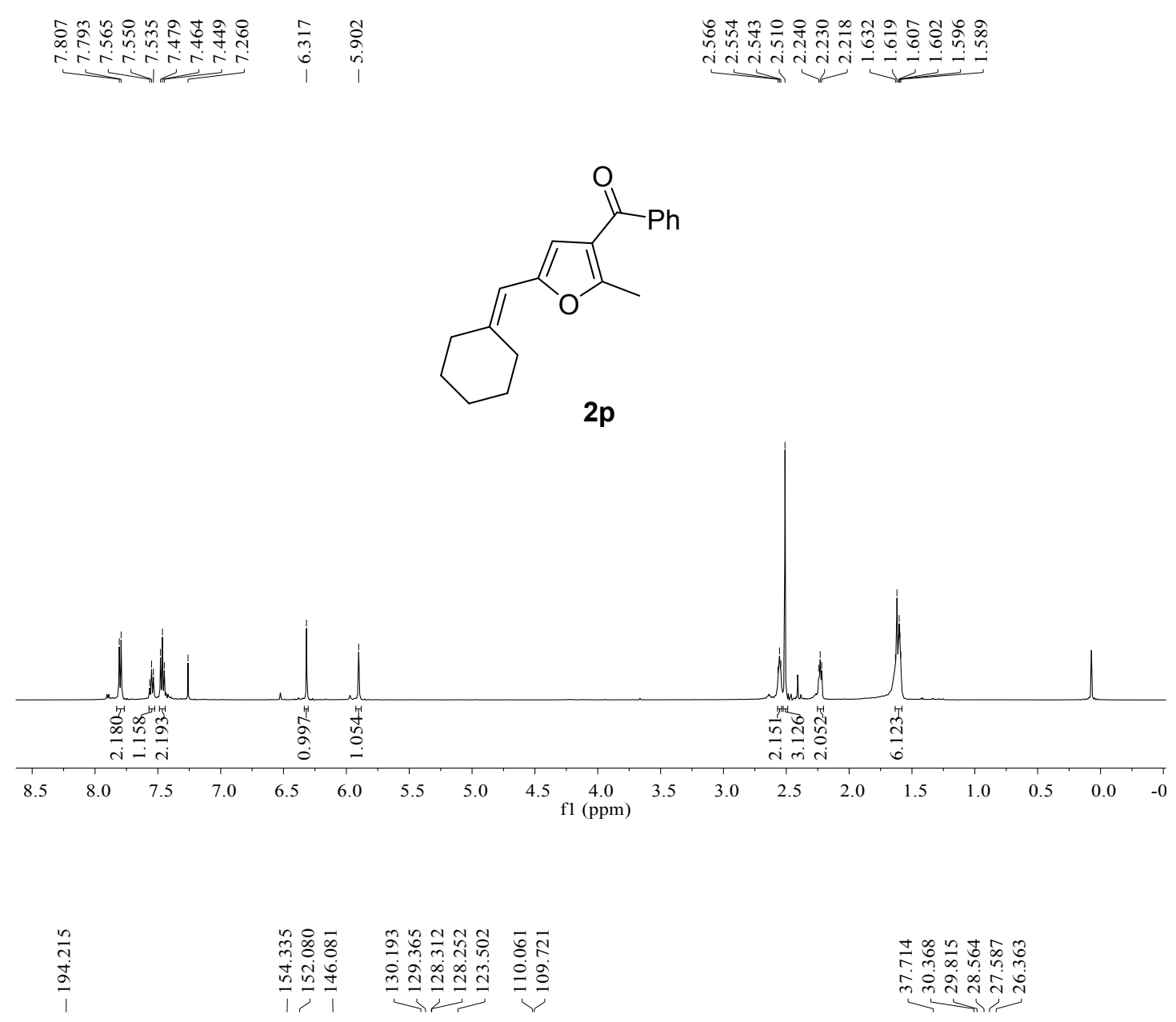

$+\frac{1}{7}$

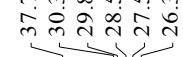

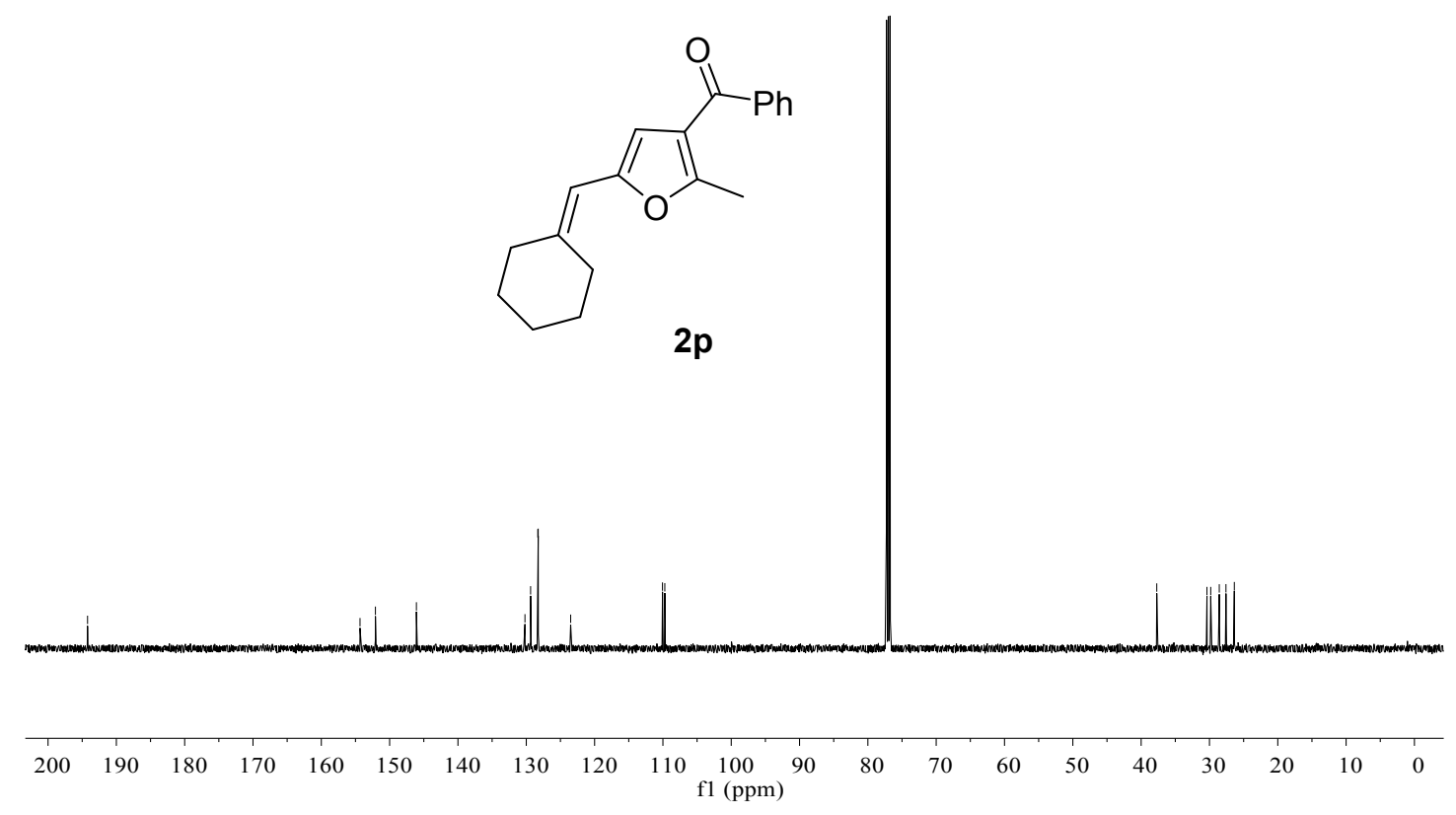




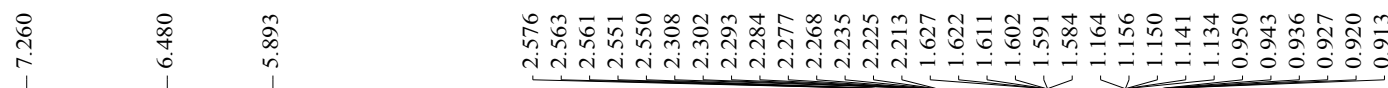

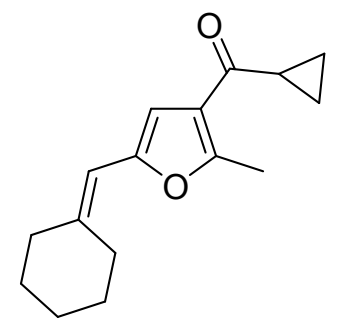

$2 q-1$

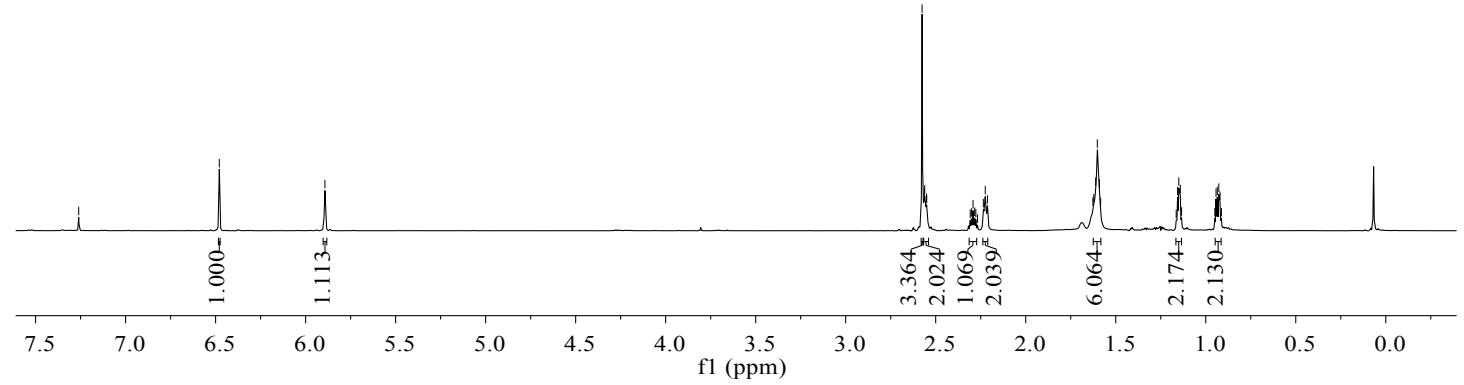

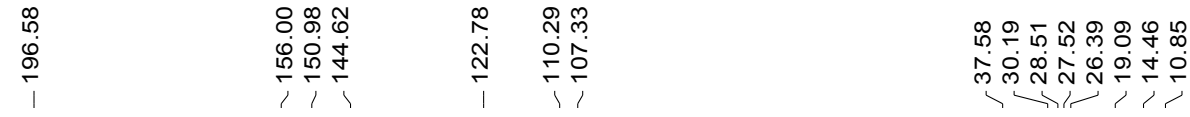

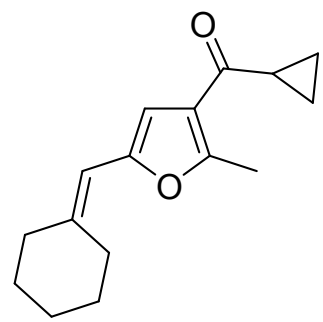

$2 q-1$

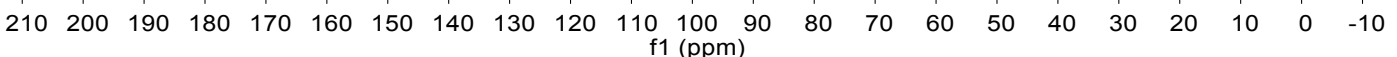



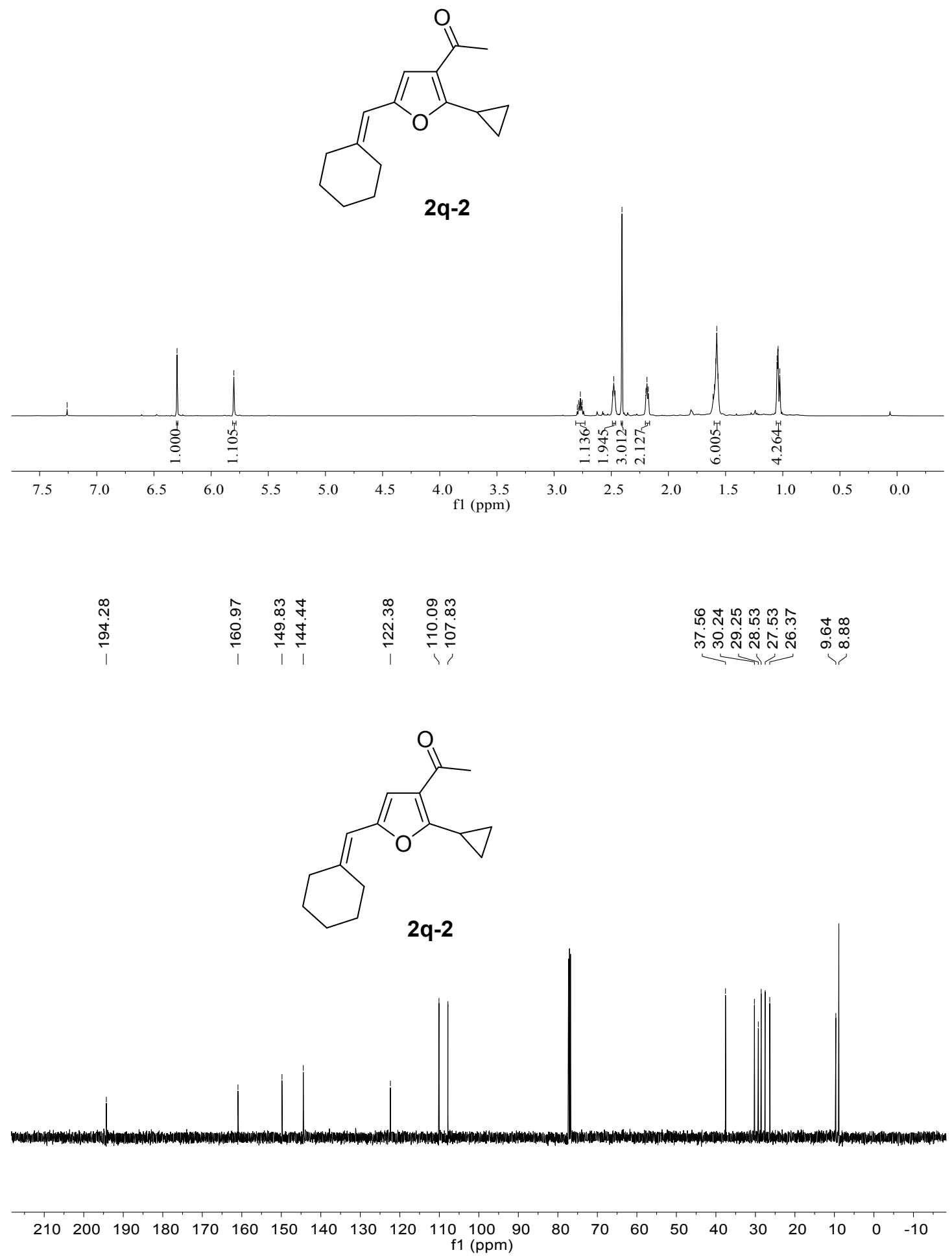

$2 q-2$ 

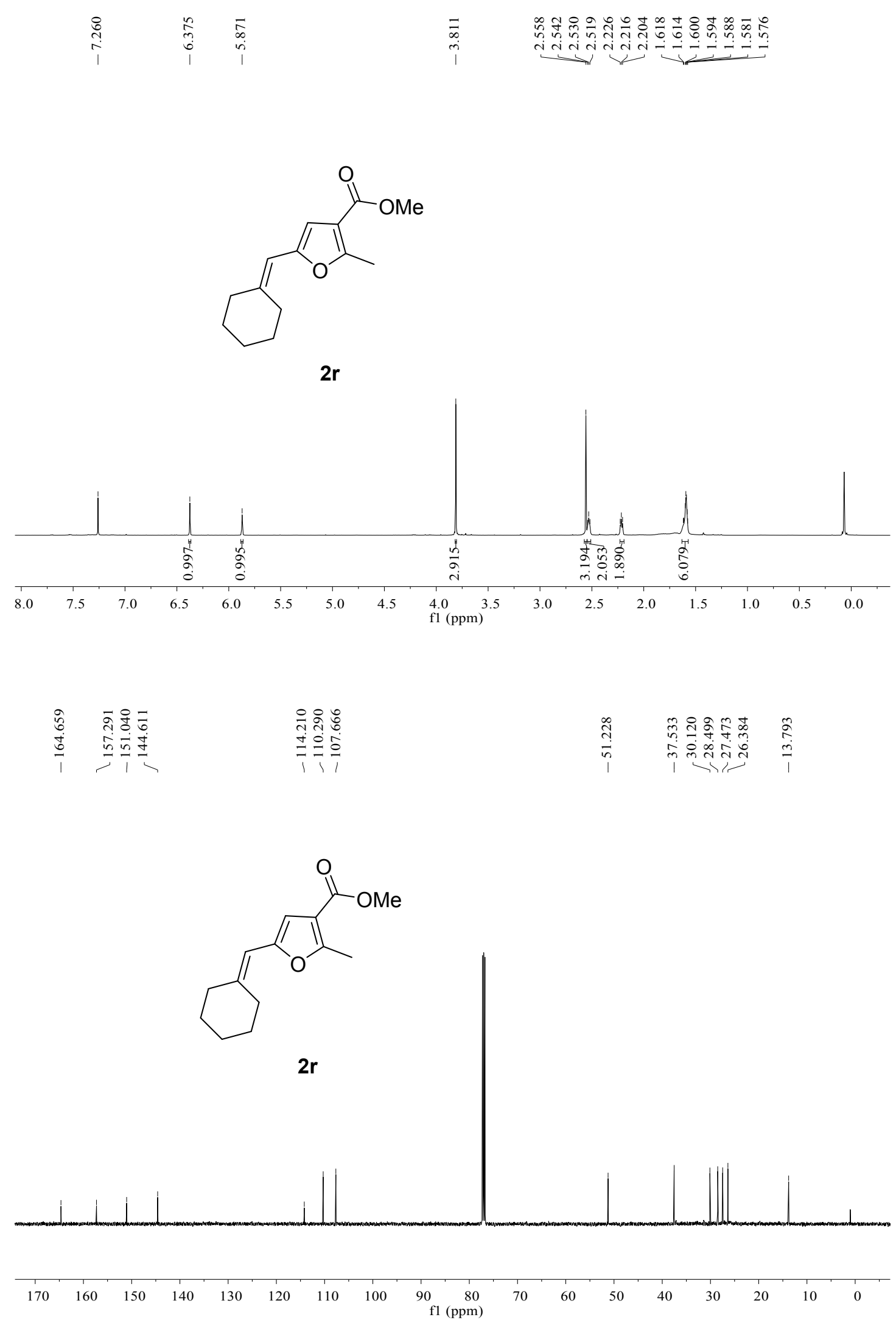


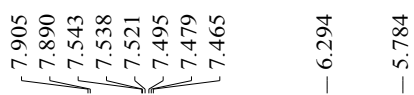

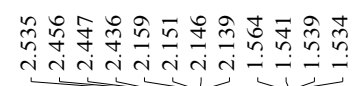

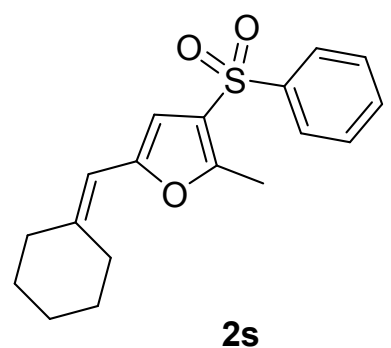

$2 s$

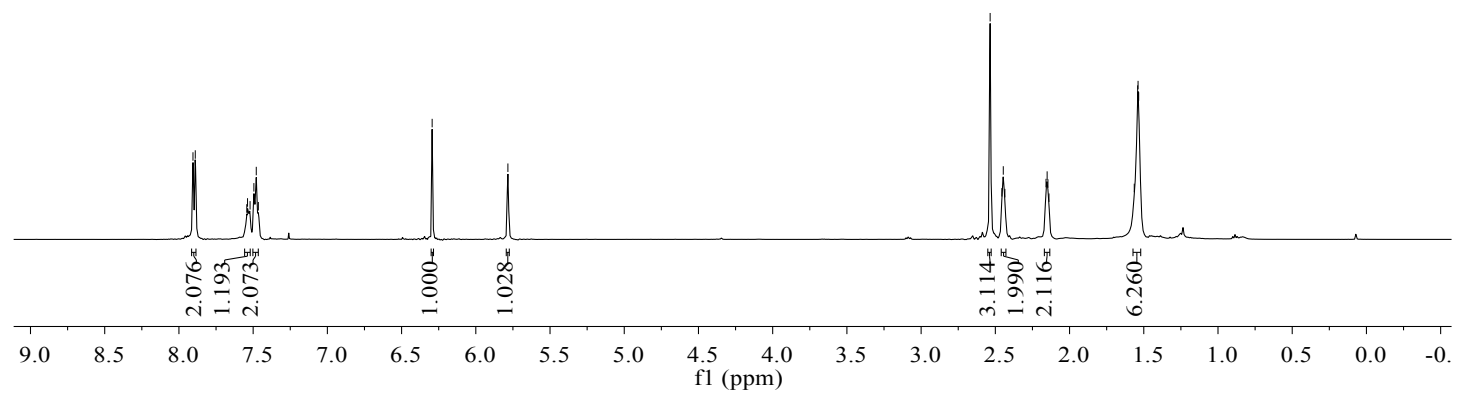

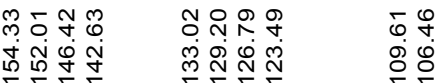

i店店店

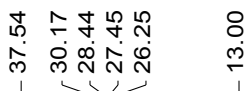<smiles>Cc1oc(C=C2CCCCC2)cc1S(=O)(=O)c1ccccc1</smiles>

$2 s$

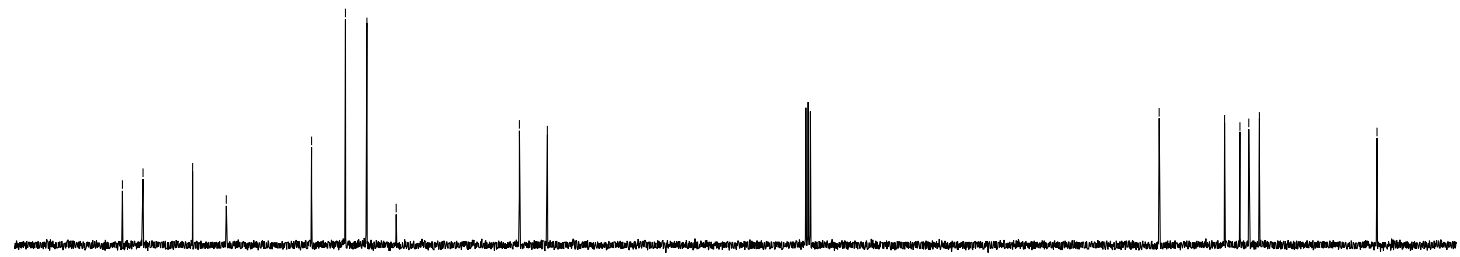

$\begin{array}{llllllllllllllll}160 & 150 & 140 & 130 & 120 & 110 & 100 & 90 & 80 & 70 & 60 & 50 & 40 & 30 & 20 & 10\end{array}$ 


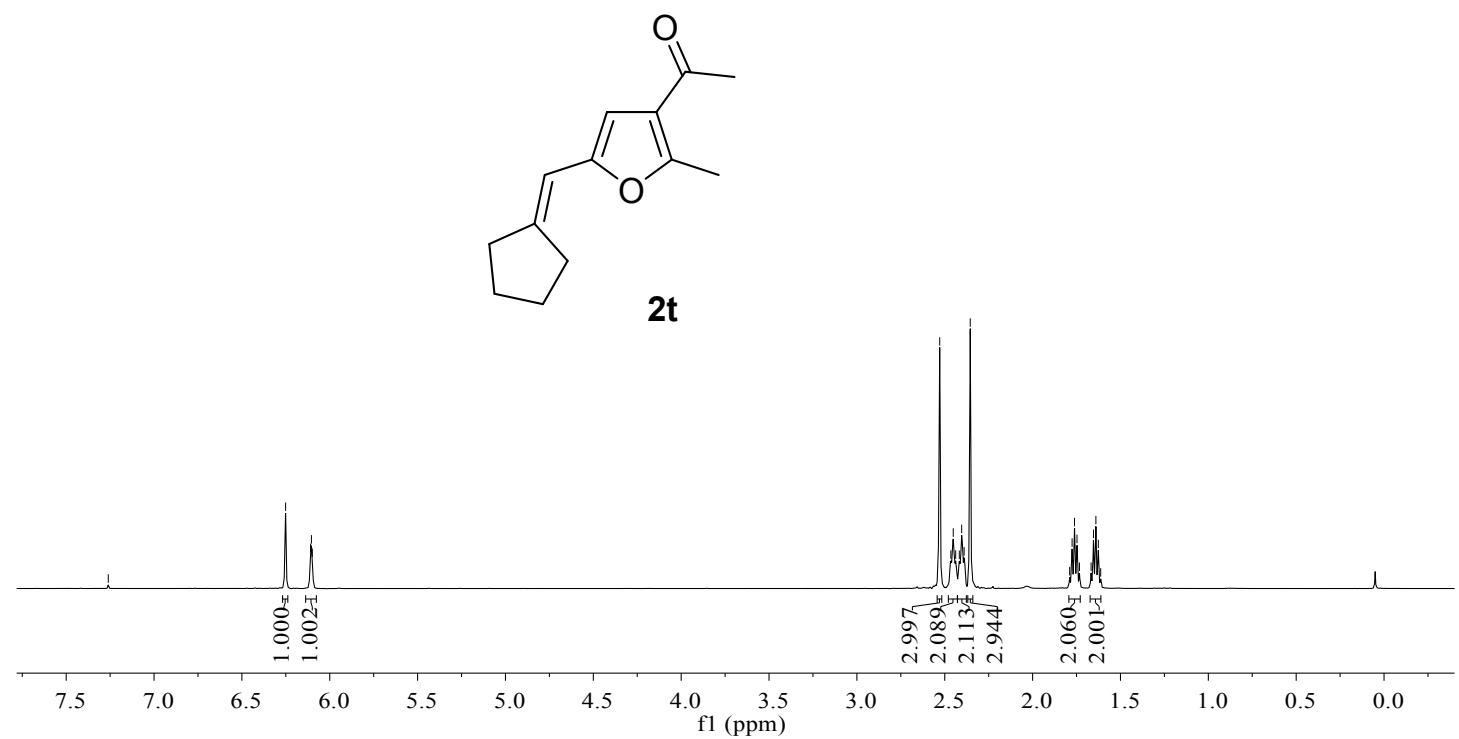

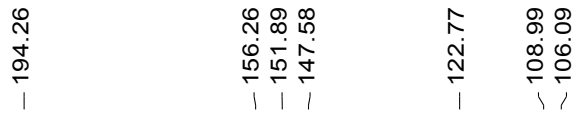

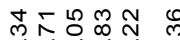

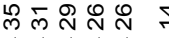

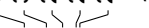
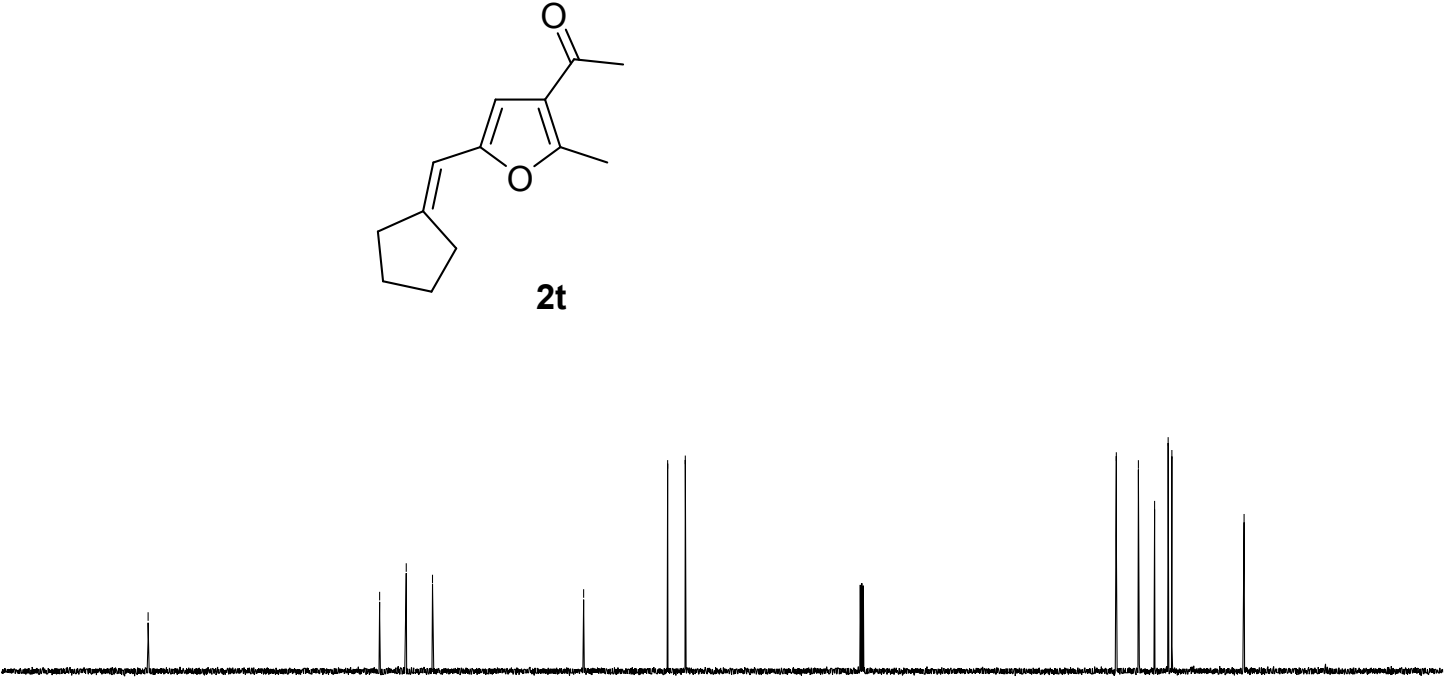

$\begin{array}{lllllllllllllllllllllll}210 & 200 & 190 & 180 & 170 & 160 & 150 & 140 & 130 & 120 & 110 & 100 & 90 & 80 & 70 & 60 & 50 & 40 & 30 & 20 & 10 & 0 & -10\end{array}$ f1 $(\mathrm{ppm})$ 


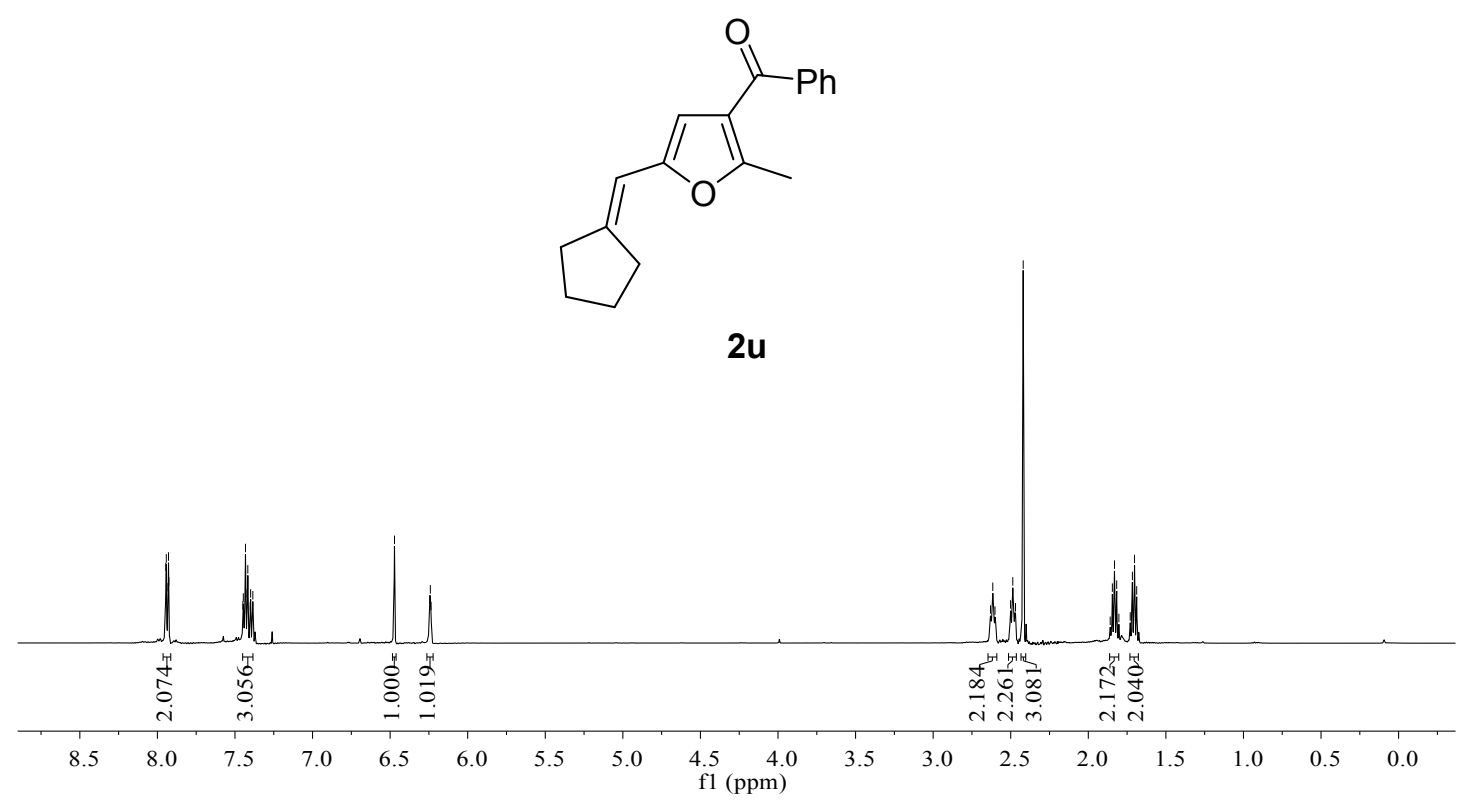

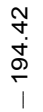

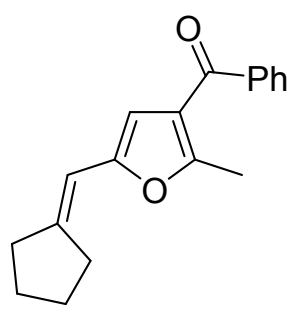

$2 u$

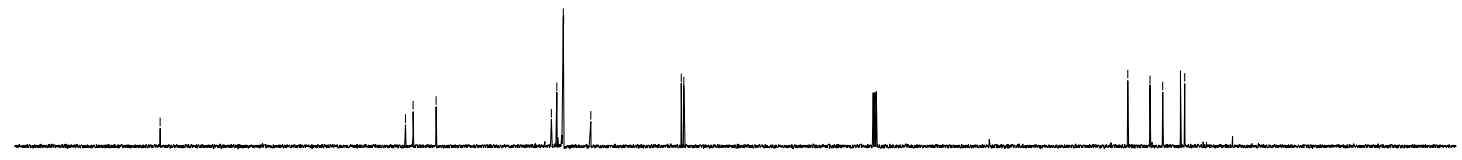

$\begin{array}{lllllllllllllllllllllll}210 & 200 & 190 & 180 & 170 & 160 & 150 & 140 & 130 & 120 & 110 & 100 & 90 & 80 & 70 & 60 & 50 & 40 & 30 & 20 & 10 & 0 & -10\end{array}$ f1 (ppm) 


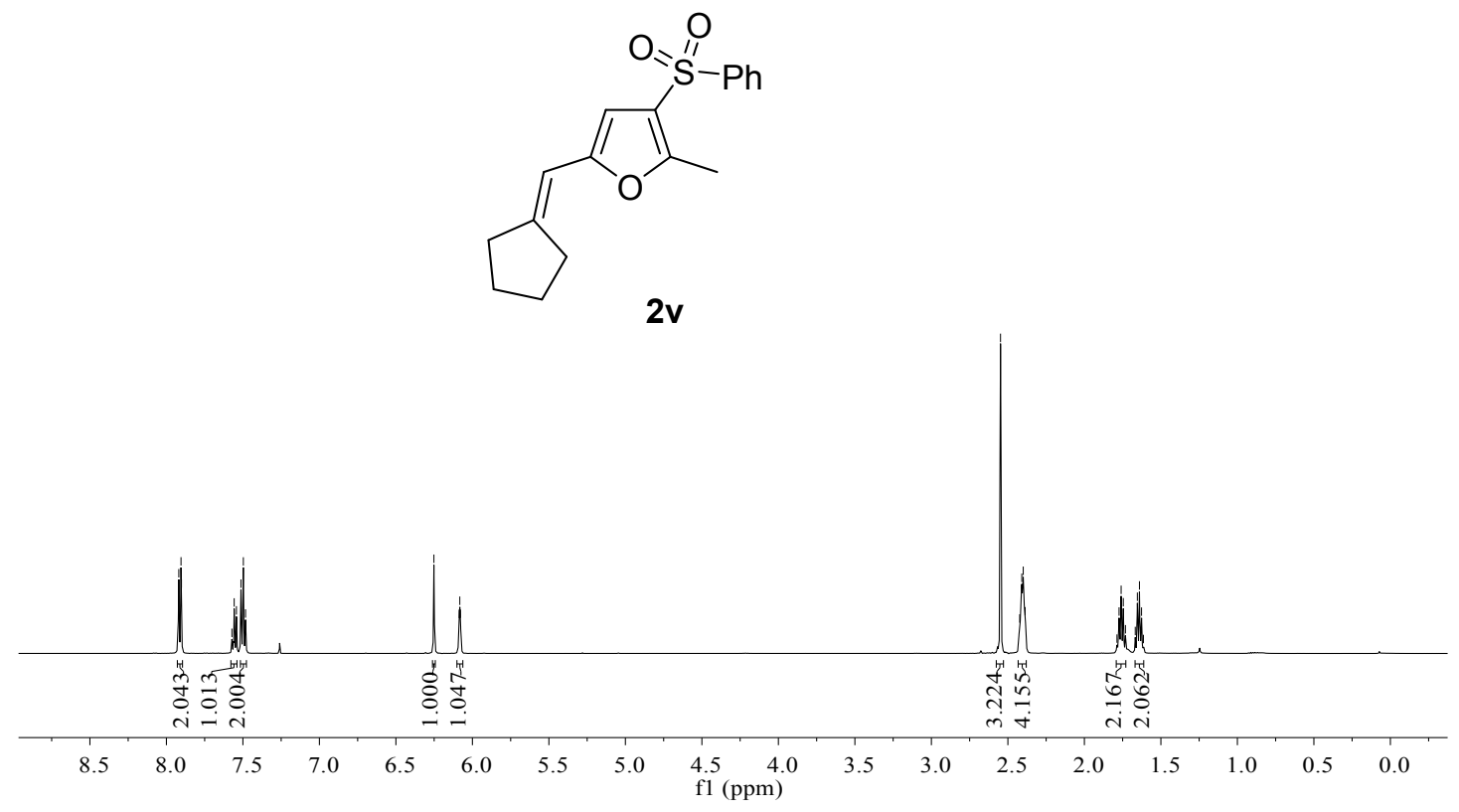

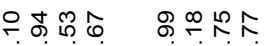

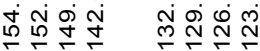
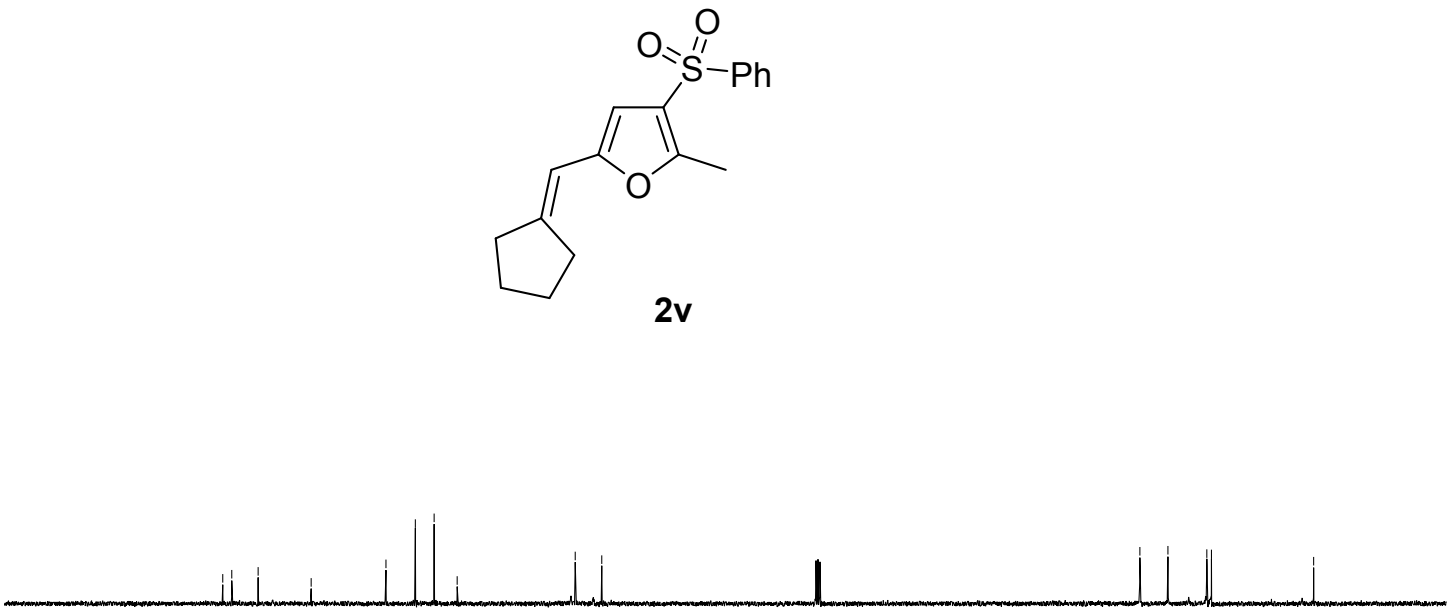

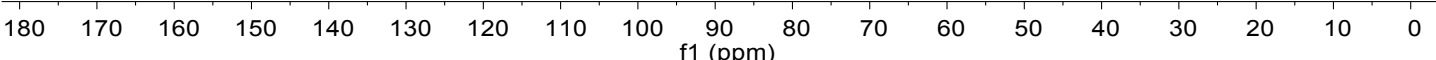



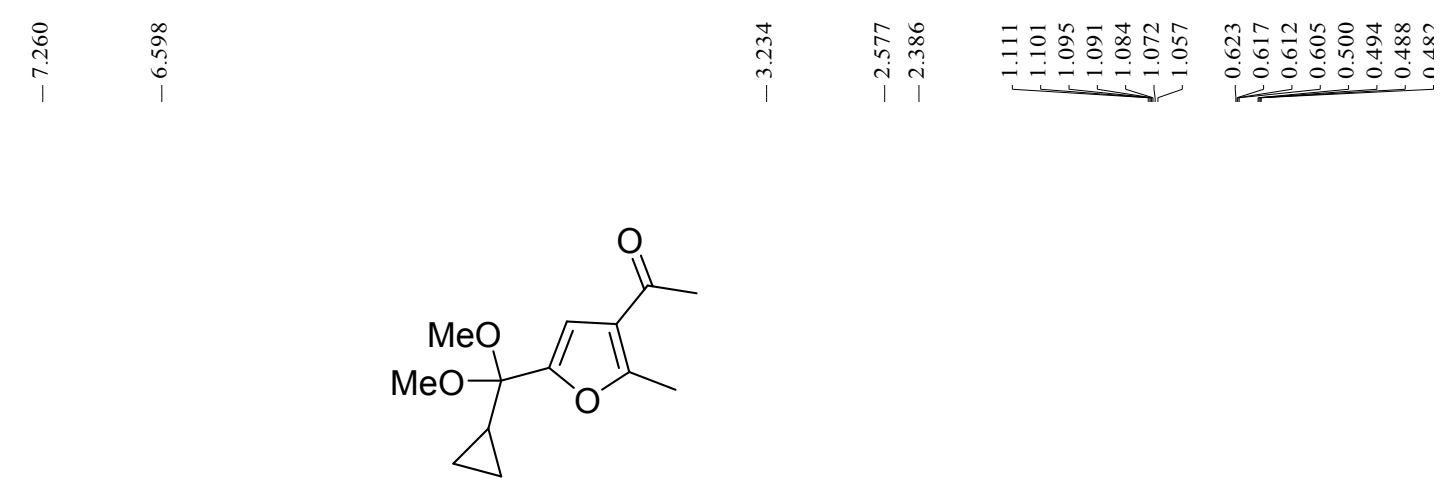

$3 a$

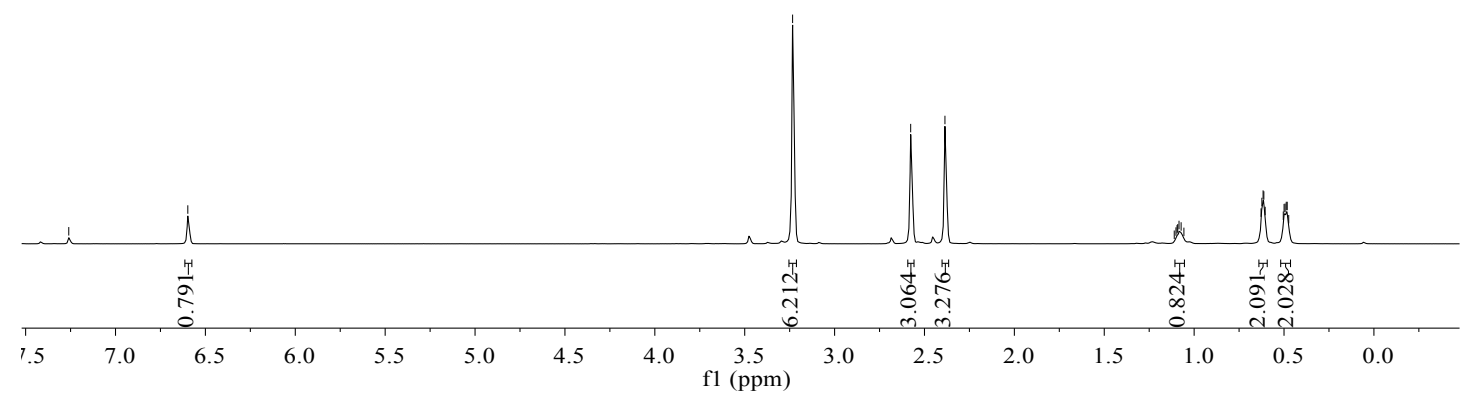

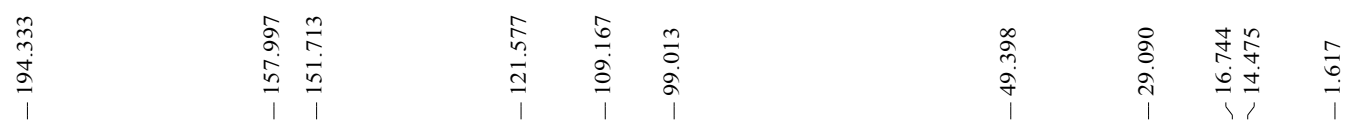

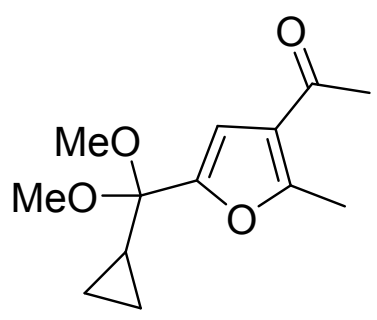

$3 a$

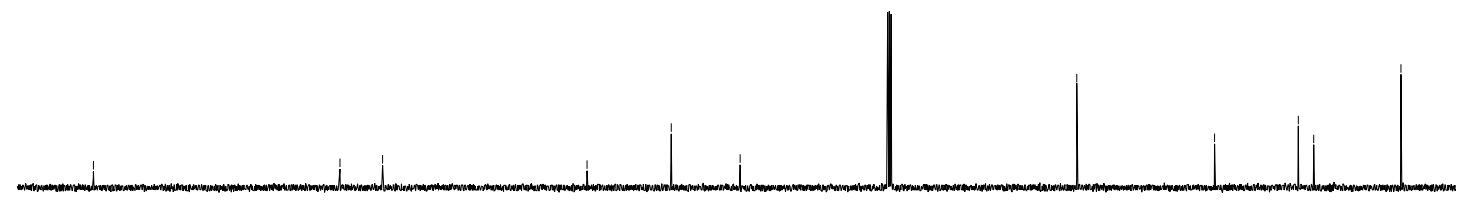

$\begin{array}{llllllllllllllllllllll}200 & 190 & 180 & 170 & 160 & 150 & 140 & 130 & 120 & 110 & 100 & 90 & 80 & 70 & 60 & 50 & 40 & 30 & 20 & 10 & 0\end{array}$ 


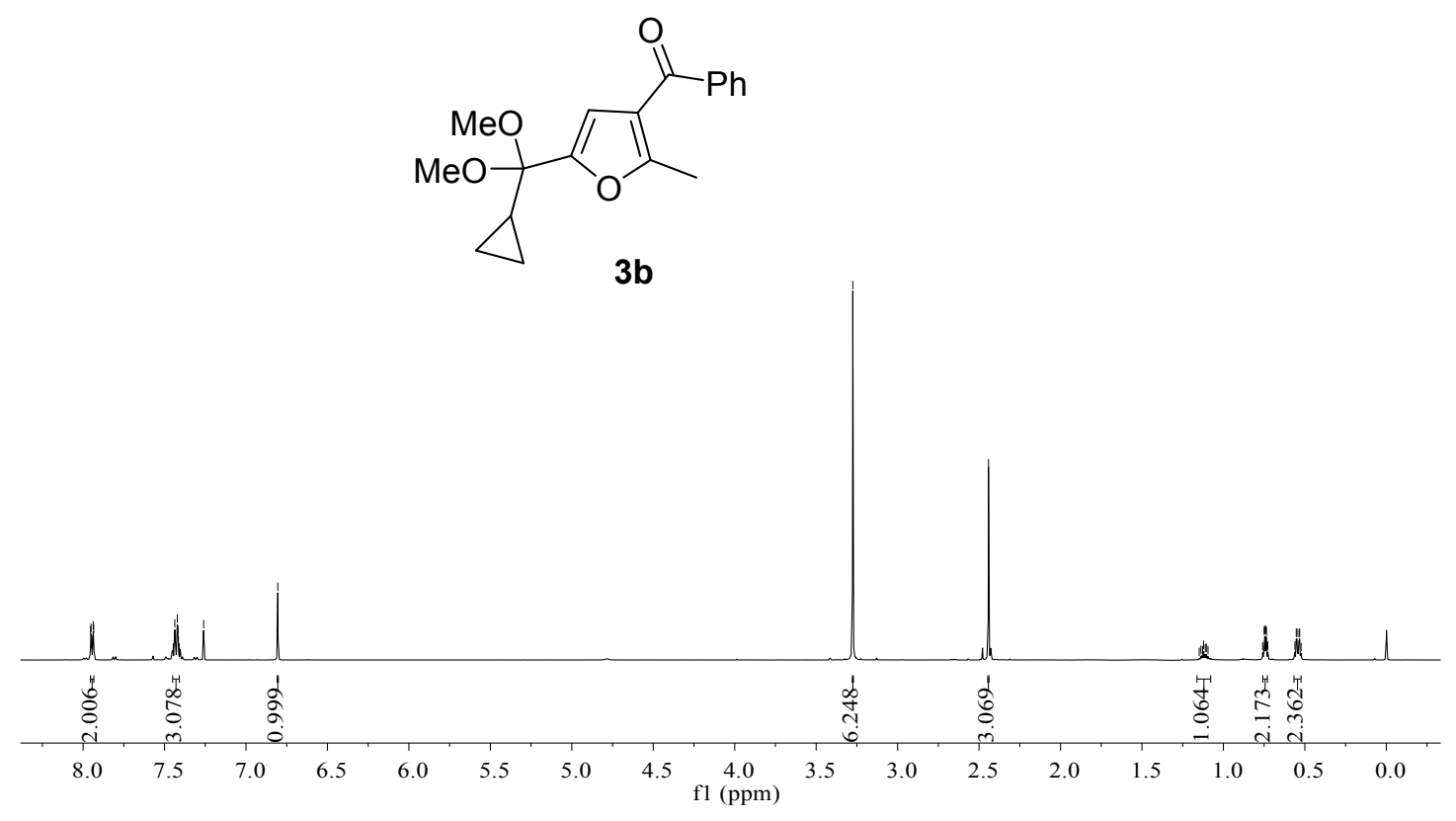

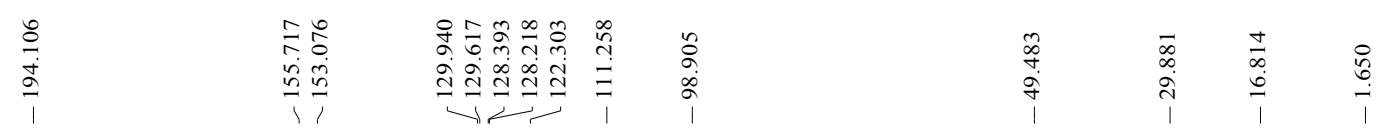

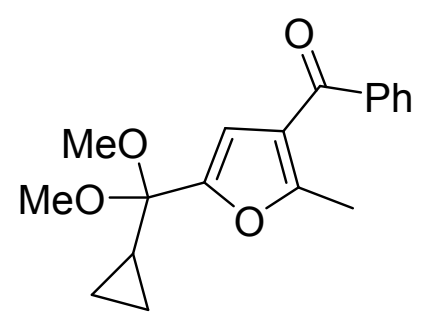

$3 b$

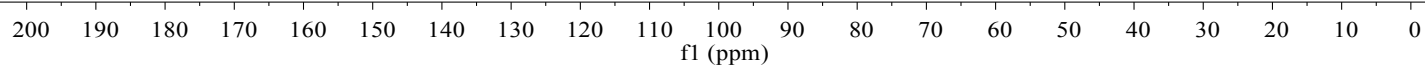




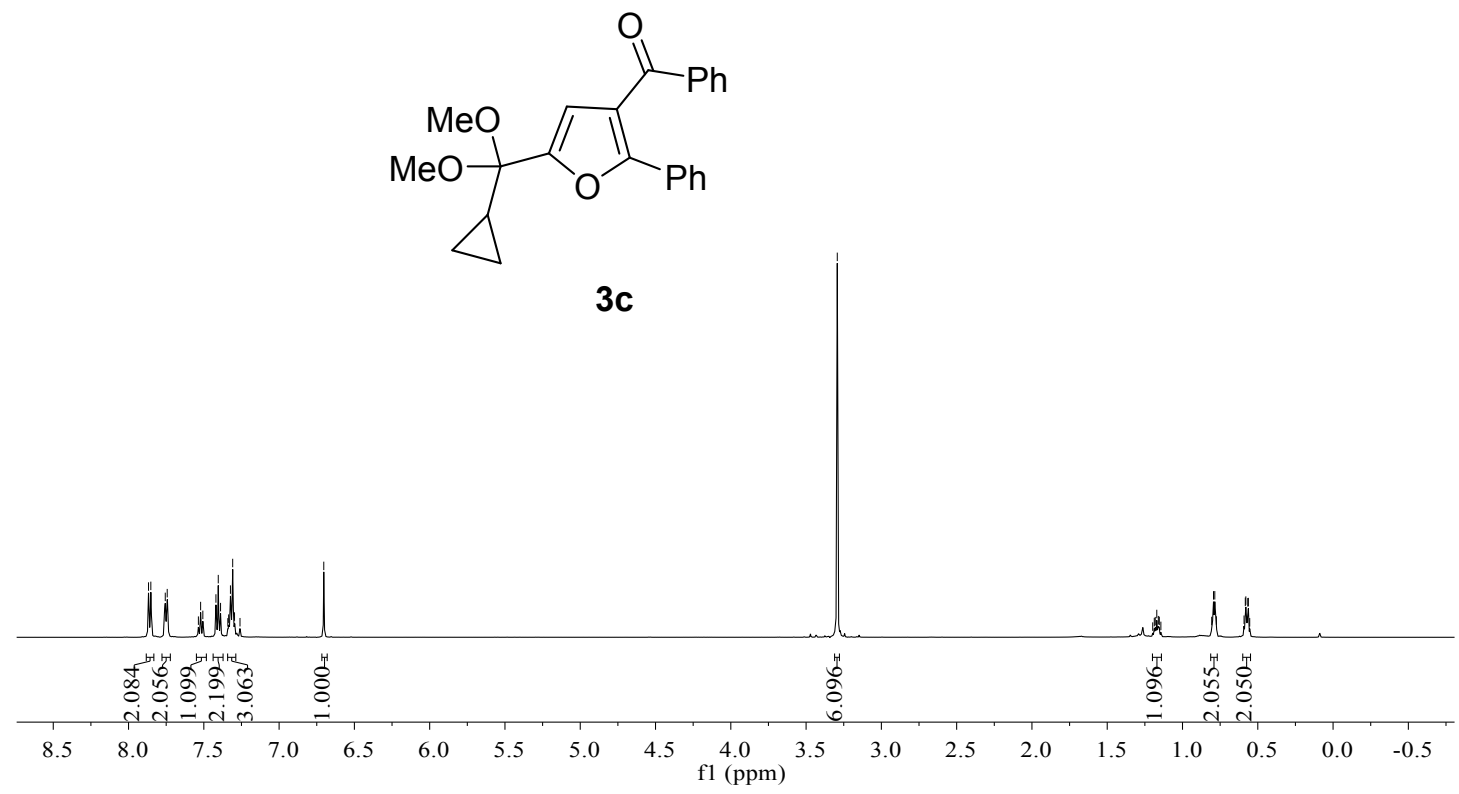

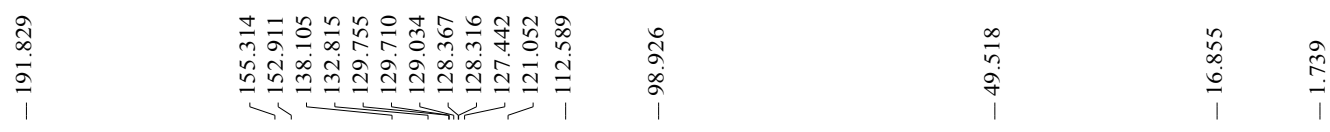
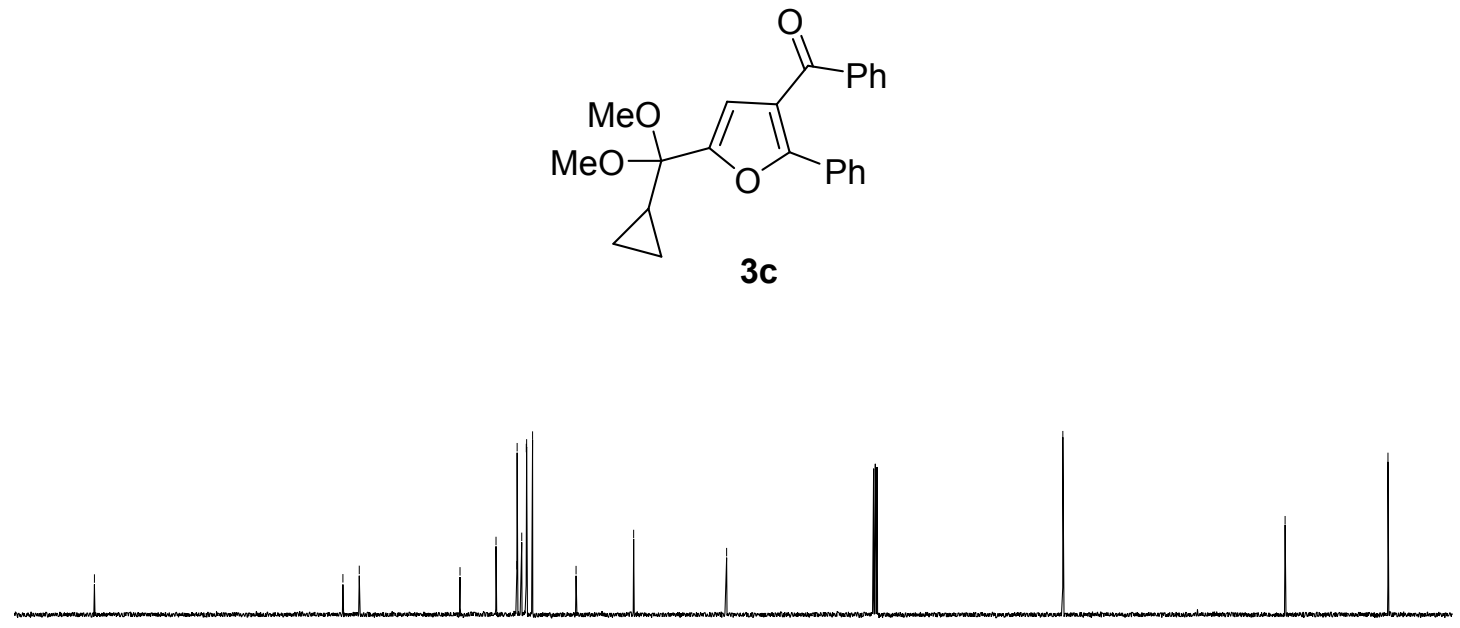

$\begin{array}{llllllllllllllllllllllllll}200 & 190 & 180 & 170 & 160 & 150 & 140 & 130 & 120 & 110 & 100 & 90 & 80 & 70 & 60 & 50 & 40 & 30 & 20 & 10 & 0\end{array}$ 